\title{
A novel type of compliant and underactuated robotic hand for dexterous grasping
}

\author{
The International Journal of \\ Robotics Research \\ 2016, Vol. 35(1-3) 161-185 \\ (C) The Author(s) 2015 \\ Reprints and permissions \\ sagepub.co.uk/journalsPermissions.nav \\ DOI: $10.1177 / 0278364915592961$ \\ ijr.sagepub.com
}

@SAGE

\author{
Raphael Deimel and Oliver Brock
}

\begin{abstract}
The usefulness and versatility of a robotic end-effector depends on the diversity of grasps it can accomplish and also on the complexity of the control methods required to achieve them. We believe that soft hands are able to provide diverse and robust grasping with low control complexity. They possess many mechanical degrees of freedom and are able to implement complex deformations. At the same time, due to the inherent compliance of soft materials, only very few of these mechanical degrees have to be controlled explicitly. Soft hands therefore may combine the best of both worlds. In this paper, we present RBO Hand 2, a highly compliant, underactuated, robust, and dexterous anthropomorphic hand. The hand is inexpensive to manufacture and the morphology can easily be adapted to specific applications. To enable efficient hand design, we derive and evaluate computational models for the mechanical properties of the hand's basic building blocks, called PneuFlex actuators. The versatility of RBO Hand 2 is evaluated by implementing the comprehensive Feix taxonomy of human grasps. The manipulator's capabilities and limits are demonstrated using the Kapandji test and grasping experiments with a variety of objects of varying weight. Furthermore, we demonstrate that the effective dimensionality of grasp postures exceeds the dimensionality of the actuation signals, illustrating that complex grasping behavior can be achieved with relatively simple control.
\end{abstract}

\section{Keywords}

Grasping, robotic hand, soft hand, human grasping, hand synergy, underactuation, pneumatics, actuator model, Pneuflex, Feix taxonomy

\section{Introduction}

Dexterous grasping is a prerequisite for task-dependent manipulation. By the term dexterous, we refer to the postural variability of the hand: the higher this variability, the more dexterous we consider a hand (for examples of grasping postures, refer to the grasp taxonomies presented in Cutkosky (1989) and Feix et al. (2009)). Such variability enables versatile grasping and manipulation: small objects can be picked up with pincer grasps, large objects with enveloping power grasps. Depending on the task, a cylindrical side grasp can be used to pick up a glass for drinking, whereas a disk grasp from above is appropriate to lift it off a cluttered table.

In robotic hands, dexterous grasping capabilities are traditionally realized through complex, multi-jointed structures and sophisticated actuation mechanisms. Such hands are expensive and difficult to design. They also require complex sensing and control. Recently, underactuated hands with passively compliant parts have become a popular alternative in robot hand design. These hands perform certain grasps robustly, have simpler mechanics, and require simpler control due to underactuation. However, there is one commonly assumed drawback of compliant hands: underactuation and passive compliance seem to render dexterous grasping difficult or even impossible. The experiments performed with our novel hand indicate the opposite.

We present a novel type of compliant and underactuated hand based on soft robotic technology. This hand, called RBO Hand 2, is capable of dexterous grasping, it is easy to build, robust to unanticipated impact, inherently safe, low cost, and easy to control. These advantages are achieved by building almost the entire hand out of soft, compliant materials or structures, rather than of rigid parts. We believe that the combination of dexterous grasping capability and ease

Berlin University of Technology, Berlin, Germany

Corresponding author:

Raphael Deimel, Berlin University of Technology, Marchstraße 23, MAR 5-1, Berlin 10587, Germany.

Email: raphael.deimel@tu-berlin.de 

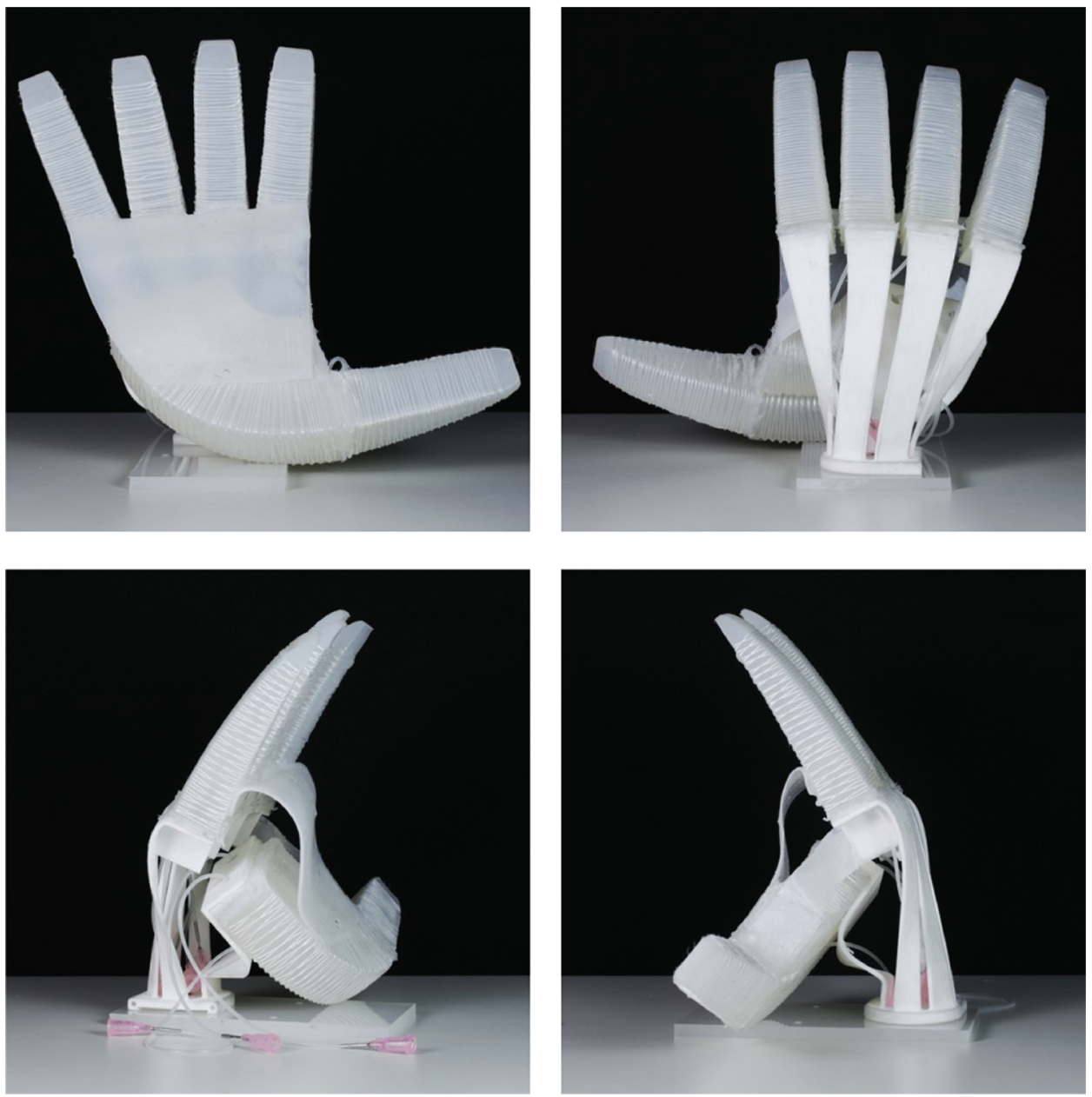

Fig. 1. The RBO Hand 2 is a compliant, underactuated robotic hand, capable of dexterous grasping. It is pneumatically actuated and made of silicone rubber, polyester fibers, and a polyamide scaffold.

of manufacturing make the presented hand well-suited for enabling novel advances in grasping and manipulation.

Our design, shown in Figure 1, purposefully maximizes the hand's passive compliance, while ensuring sufficient structural support to lift objects. We believe that this design choice is critical for robust grasping: First, passive compliance facilitates obtaining force closure in power grasps (Deimel and Brock, 2013; Dollar and Howe, 2010). Second, passive compliance facilitates the use of contact with the environment to aid attaining a grasp. This strategy, the exploitation of environmental contact to reduce uncertainty, has been shown to increase grasp performance in humans as well as robots (Deimel et al., 2013; Kazemi et al., 2014). For these reasons, passive compliance is a key ingredient for robust grasping. In this paper, we want to show that in addition to the two aforementioned advantages, passively compliant hands can also perform dexterous grasping. Indeed, our results indicate that passive compliance even facilitates dexterous grasping.

An opposable thumb is important to achieve dexterity in human and robotic hands. We evaluate the thumb dexterity of the RBO Hand 2 using the Kapandji test (Kapandji, 1986). This test is commonly used to evaluate thumb dexterity in human hands after surgery. In addition, we show that the hand is capable of enacting 31 out of 33 grasp postures of the human hand from the comprehensive Feix taxonomy (Feix et al., 2009). We evaluate the space of hand posture exhibited by humans and the RBO Hand 2 which we find to be similar. We also show that four actuation degrees of freedom suffice to achieve a postural space with more than these four dimensions. This implies that the variability in grasping posture is only partially generated by the hand's actuation. The remaining variability is the result of interactions between hand and object. These interactions, we claim, are greatly simplified and enriched by the extensive use of passive compliance in the hand's design. These results indicate that dexterous grasping is easier to achieve with passively compliant than with traditional, stiff-linked hands.

This paper extends our previous work (Deimel and Brock, 2014) in several important ways. In Section 6.3, we extend the analysis of the hand's dexterity by comparing its postural diversity to that of the human hand, given a set of diverse grasps. This is important, as it provides support for the statement that compliance enhances dexterity and ensures that the RBO Hand 2 has as diverse postures as the 
human counterpart. We also present and validate a novel model for the actuator's behavior in Appendix B. This enables the validation of novel actuator designs prior to manufacturing. Furthermore, we significantly extended the description of the hand design and production process in Section 4. Finally, we publish the experimental data sets, videos, and high resolution images of the human and robotic experiments related to the Feix taxonomy in Extensions 1-6.

\section{Related work}

Many highly capable robotic hands exist. A historical overview, surveying robotic hands from over five decades, provides an excellent overview (Controzzi et al., 2014). An analysis of robot hand designs with respect to grasping capabilities was recently presented by Grebenstein (2012). As the notion of compliance is central to our hand design, we will limit our discussion to hand designs that deliberately include this concept.

We distinguish between two types of hands, actively and passively compliant. The former can be achieved by using active control on fully actuated or even hyper-actuated systems, where every degree of freedom can be controlled. Examples are the impressive Awiwi hand (Grebenstein, 2012), the ShadowRobot Shadow Dexterous Hand, and the SimLab Allegro Hand (Bae et al., 2012). These hands achieve dexterity and compliance through fast and accurate control, which comes at the price of mechanical and computational complexity. As a result, these hands tend to be mechanically complex and expensive to manufacture. Mechanical complexity can also increase the probability of hardware failure.

The alternative is to make hands passively compliant by including elastic or flexible materials. Building a passively compliant joint is much cheaper than building an actively controlled one in terms of costs, spatial volume, and mechanical complexity. Passive compliance limits impact forces, a crucial property for an end-effector designed to establish contact with the world. More degrees of freedom can better adapt to the shape of an object greatly enhances grasp success and grasp quality. At the same time, the hand can be underactuated, effectively offloading control to the physical embodiment of the hand.

A pioneering work in grasping with passive compliance was the soft gripper by Hirose and Umetani (1978). Recently, a whole range of grippers and hands were built using passive compliance: the FRH-4 hand (Gaiser et al., 2008), the SDM hand and its successor (Dollar and Howe, 2008; Ma et al., 2013; Odhner et al., 2014), the starfish gripper (Ilievski et al., 2011), the THE Second Hand and the Pisa-IIT Soft Hand (Catalano et al., 2014), the ISRSoftHand (Tavakoli and Almeida, 2014), the Positive Pressure Gripper (Amend et al., 2012), the RBO Hand (Deimel and Brock, 2013), and the Velo Gripper (Ciocarlie et al., 2013). A different source of inspiration was taken by
Giannaccini et al. (2014), who built a compliant gripper inspired by the octopus arm.

The practical realization of underactuated hands is matched by theoretical approaches to analyze and evaluate their dexterity (Gabiccini et al., 2013; Prattichizzo et al., 2012). The most promising models rely on the hypothesis of a low-dimensional representation of grasp postures, called synergies (Santello et al., 1998). Odhner et al. (2014) simplify the underactuated hand mechanics into compliance ellipsoids at possible locations of contact points. However, these approaches require accurate knowledge of grasp posture, contact point locations and contact forces. Given current sensor technologies, this information is difficult to obtain. Interestingly, humans are able to grasp under comparable conditions with strongly impaired perception, e.g. with blurred sight and wearing a glove (Deimel et al., 2013). This suggests that there is an alternative, perceptually less demanding representation of compliant behavior.

The inclusion of compliance into the design of robotic hands has led to significant improvements in performing power grasps. Very little work has examined the effect of compliance and underactuation on the dexterity of a robotic hand. Closing this gap will be the focus of this paper. Tavakoli et al. (2014) recently characterized the influence of reducing the number of actuated degrees of freedom on the number of possible grasps for the ISR-SoftHand. This anthropomorphic hand relies on extensive compliance using elastomeric joints and deformable finger pads. They found that about four to six actuated degrees of freedom are enough to enact a broad set of human grasps and that an opposable thumb is crucial to achieve this, which corroborates our own findings.

\section{PneuFlex actuators}

The RBO Hand 2 uses a highly compliant, pneumatic continuum actuator design, called PneuFlex, which was first presented in Deimel and Brock (2013). PneuFlex actuators can be manufactured within a day and use materials that are cheap and non-toxic. Figure 2 illustrates the working principle. When inflating the contained chamber with air the pressure forces the hull to elongate along the actuator. The bottom side contains an inelastic fabric to prohibit elongation. This causes a difference in length between the top and bottom side and the actuator bends. Radial fibers stabilize the actuator's shape and greatly increase the attainable curvature.

\subsection{Manufacturing actuators}

A distinguishing feature of the PneuFlex actuator is the integrated design pipeline, which enables rapid prototyping of actuators with widely varying properties without changing the production process. The steps of the process are illustrated in Figure 3.

To create an actuator from scratch, first a set of planes is defined along a line or curve, on which the local cross 


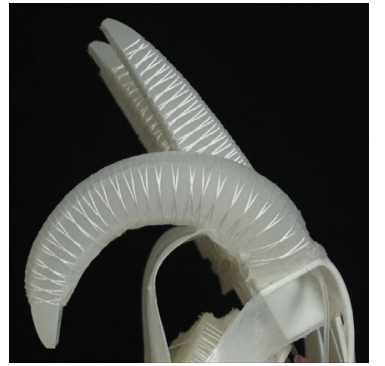

(a) An inflated Pneu Flex actuatorin front of deflated ones.

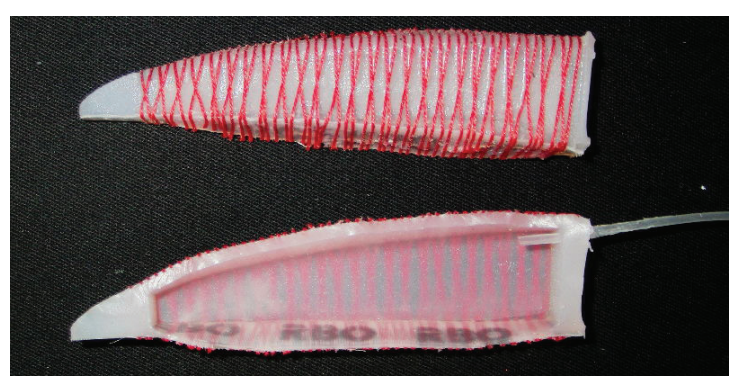

(b) Cut of a PneuFlex continuum actuator.

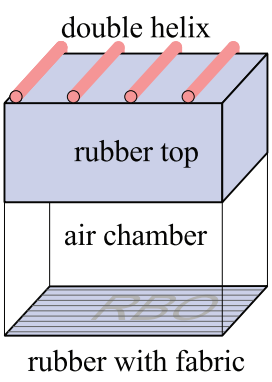

(c) Functional parts

Fig. 2. Working principle and structure of a PneuFlex actuator: When inflated, the top of the finger, consisting of translucent silicone, extends, thereby bending as its motion is constrained by the bottom of the finger, into which an inelastic fabric is embedded. The helically wound threads stabilize the actuator shape and relieve the rubber of non-functional strains, i.e. the inflation leads to bending rather than to radial expansion.

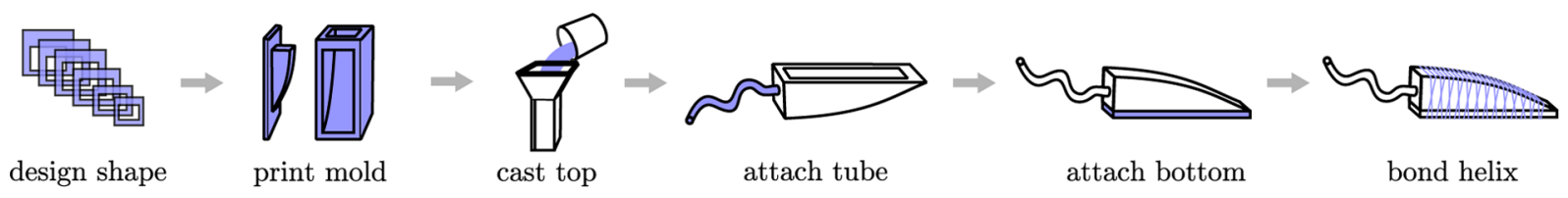

Fig. 3. Production steps for making a PneuFlex actuator with a custom stiffness and actuation ratio profile.

section of the actuator is defined. The principal shape parameters for each cross-section (height, width, hull thickness) are determined from the desired actuation ratio and stiffness at each point along the actuator, e.g. by using the model provided in Appendix B.

In the second step, the set of cross-sections is translated into a 3D model of a two-part mold for casting the rubber body of the actuator. The model is produced on a 3D printer. Because we need to separate the mold from the cast, the bottom side of the actuator is not included.

In the next step, the top part is cast using the printed mold and addition-cure silicone.

After unmolding, a silicone tube is inserted at a convenient position into the top part and bonded to it. The tube enables us to easily connect the actuator to the pneumatic control.

Afterwards the air chamber is closed by placing the top part on a thin $(1-2 \mathrm{~mm})$ sheet of freshly cast silicone that embeds a bendable but inextensible PET fabric.

When the bottom layer has cured, a sewing thread is wound around the actuator in form of a double helix. To fixate the thread in place, a thin layer of addition-cure silicone is applied to the top and bottom side. This step finishes the actuator.

The presented process enables us to freely change width and height of the actuator and the thickness of the rubber hull. We can also create straight as well as curved actuators, as long as the bottom layer stays in a plane.

The actuator design space can be explored by varying shape and size of the actuator, and by varying the thickness

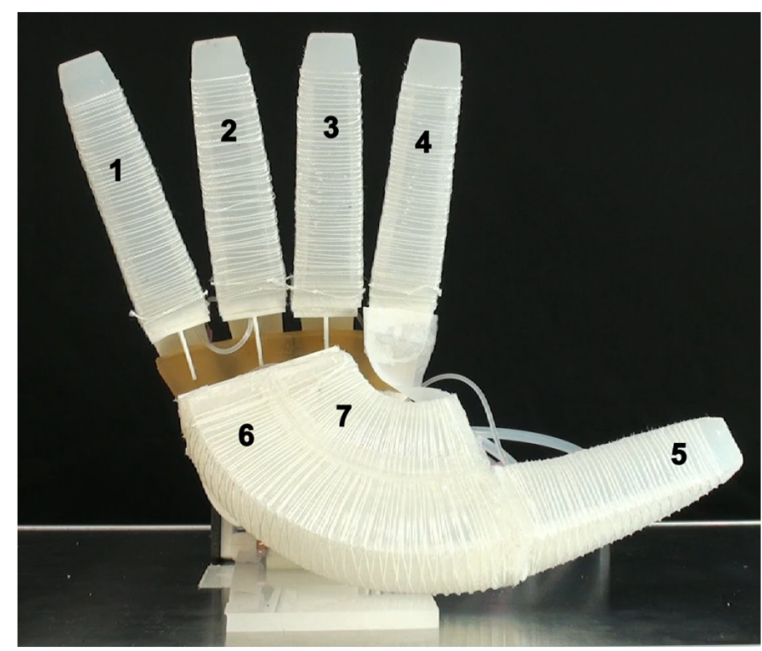

Fig. 4. The seven actuators of the soft anthropomorphic hand: four fingers (1-4), thumb (5), and the palm, consisting of two actuators $(6,7)$.

of the silicone hull at the top, side and bottom. In addition, available silicone types let us vary the shear modulus by an order of magnitude. All of these parameters affect the bending behavior, stiffness, and limits of the actuator. If needed, a bellows-shaped hull extends the design space towards larger curvatures and lower stiffnesses. Curved actuators can realize 3D motion. For example, the two palm actuators (actuators 6 and 7 in Figure 4) are used to achieve thumb opposition. Differential inflation of the two actuators provides additional dexterity. 
Actuators can also be packaged together, similarly to muscle fibers, allowing for redundant actuation or variation of the actuation strength. Joining actuators also enables the implementation of multiple deformation modes or the deliberate mixing of deformation modes (Bishop-Moser et al., 2012).

In addition their flexible production process, PneuFlex actuators are robust to impact and blunt collisions, are inherently safe, and are not affected by dirt, dust, or liquids. However, they can easily be cut or pierced.

\subsection{Modeling actuators}

The PneuFlex actuator shares many properties with other recently published continuum actuators, most notably the fast PneuNet actuators (Mosadegh et al., 2014) and the actuator by Galloway et al. (2013). In contrast to these actuator designs, the PneuFlex design and production process is optimized to provide freely adaptable cross-sections which determine actuation ratio and stiffness, the ability to include multiple separate air chambers, and to provide access to its internal space for the integration of sensors and wiring.

We provide a detailed analysis of basic actuator behavior in Appendix B and also propose design rules for successful actuator design in Section B.11. Additional insights can be drawn from related research on continuum actuators. For example, Bishop-Moser et al. (2012) characterize all basic motions attainable by changing inclinations of the reinforcement helices. Others proposed approximate numeric models based on twisted, one-dimensional beams (Giorelli et al., 2012; Renda et al., 2012).

\section{Hand design}

In this section, we describe the components of our soft anthropomorphic hand (RBO Hand 2, see Figure 1). The entire hand weighs $178 \mathrm{~g}$ and can carry a payload of up to $0.5 \mathrm{~kg}$. Higher payload can easily be achieved, if necessary, as we will explain in Section 7.

\subsection{Morphology}

The design space of possible hands is very large. For this hand, we chose an anthropomorphic design in shape and size for three reasons. First, we know the human hand form enables dexterous grasping in humans. By starting with a human-hand-like morphology, we start with a proven hand design. Second, many objects have been built for manipulation by a human hand and match the anthropomorphic form factor. Third, we can use well-established grasp taxonomies and compare our designs with humans and many other robotic hands.

\subsection{Control}

Pneumatic control of the PneuFlex actuators is based on a simple linear forward model for computing valve opening

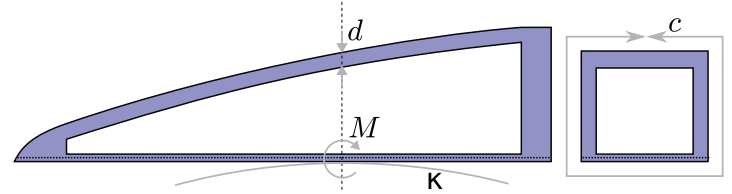

Fig. 5. Illustration of the finger geometry and its principal parameters: $\kappa$ (local curvature), $M$ (moment around the passive layer at the interface), $d$ (top side rubber thickness) and $c$ (circumference).

times. The model takes into account the regulated supply pressure to achieve a desired channel pressure which corresponds to a desired bending radius or contact force. Alternatives to this digital control are cylinder-based continuous control systems (Marchese et al., 2014). Renda et al. (2012) demonstrate a computationally simple forward model for an artificial octopus arm. This model can also be used for PneuFlex actuators.

For the experiments, control was implemented with industry-grade air valves and a separate air supply. For a mobile system, the control system can easily be optimized for size and weight, because the required air flows are an order of magnitude smaller than provided by industrial grade valves. For the same reason, pressurized air can be effectively sourced by small compressors and small tanks. In systems where electric energy is scarce, high-pressure tanks can provide storage of air with high energy density (Wehner et al., 2014).

\subsection{Fingers}

The five fingers of our hand are single PneuFlex actuators (see Figure 4). The index, middle, ring, and little finger are $90 \mathrm{~mm}$ long and of identical shape, the thumb actuator is $70 \mathrm{~mm}$ long. All fingers get narrower and flatter towards the finger tip. By using actuators as fingers, we can exploit the excellent compliance and robustness of the actuators and greatly simplify the design.

The mechanical behavior of the finger can be described by the local curvature $\kappa$ and torsional stiffness $M$ around the bottom side for short segments of the actuator and is determined by the geometry of the actuator cross-section (see Figure 5). Appendix B contains an analysis of this simplified model of the actuator, which provides surprisingly simple rules to design the ratio of curvature $\kappa$ to pressure $p$ at each segment along the actuator by varying the hull thickness $d$ :

$$
\frac{\Delta \kappa}{\Delta p} \approx \frac{1}{G \cdot d}
$$

where $G$ is the shear modulus of the rubber and constant within an actuator.

Translational forces between actuator segments are mainly transmitted by the inelastic fabric of the bottom layer and therefore do not need to be considered. The model 
in Appendix B also yields a rule of thumb for the torsional stiffness of an actuator segment. For an approximately squared cross-section, stiffness scales with circumference $c$ :

$$
\frac{\Delta M}{\Delta \kappa} \approx G \cdot d \cdot c^{4}
$$

As an example, for the fingers of the hand we chose to increase stiffness linearly from tip to base and keep the actuation ratio constant. Such a profile has also been used in the Soft Gripper (Hirose and Umetani, 1978). Using $x$ as the distance from the tip along the actuator, the crosssection at this point is defined by

$$
\begin{aligned}
& d(x)=\text { const } \\
& c(x) \propto x^{\frac{1}{3}}
\end{aligned}
$$

The resulting geometry of the actuator is illustrated in Figure 5. According to our model, the aspect ratio of the cross section does not strongly influence stiffness and actuation ratio. So to give the finger a visually more appealing shape, we set the width to

$$
\operatorname{width}(x) \propto x^{\frac{1}{8}}
$$

\subsection{Palm}

A key feature of the human hand is the opposable thumb. We realize it in our hand by actuating the palm (see Figure 4). The palmar actuator compound consists of two connected actuators. Its base shape is a circular section of $90^{\circ}$ with $78 \mathrm{~mm}$ outer and $25 \mathrm{~mm}$ inner radius. The actuator curves perpendicular to the passive layer. The stiffness as well as the actuation ratio remain constant along the curved actuator. They are also designed to be twice as stiff as the fingers to account for the fact that the two actuators in the palm oppose four fingers. Figure 8 provides an impression of the possible thumb motions when the two palm actuators are inflated either together or differentially.

In addition to enabling thumb opposition, the palm also provides a compliant surface that, together with the fingers, is used to enclose objects in various power grasps. To augment this function, the fingers and the palmar actuator are connected by a thin sheet of fiber-reinforced silicone, covering the gap between palm actuators and fingers (shown in Figure 1, but absent in Figure 4). This sheet transmits tensile forces between fingers and palm, and between adjacent fingers. This stabilizes the underlying scaffold during power grasps or for heavy loads, as shown in Figure 11.

\subsection{Thumb}

Like the other fingers, the thumb consists of a single PneuFlex actuator. The thumb is shorter and twice as stiff, but also features a linear stiffness profile. A faithful imitation of how humans use their thumb would require a negative curvature close to the tip, as shown in Figure 6, and would significantly increase complexity of manufacturing
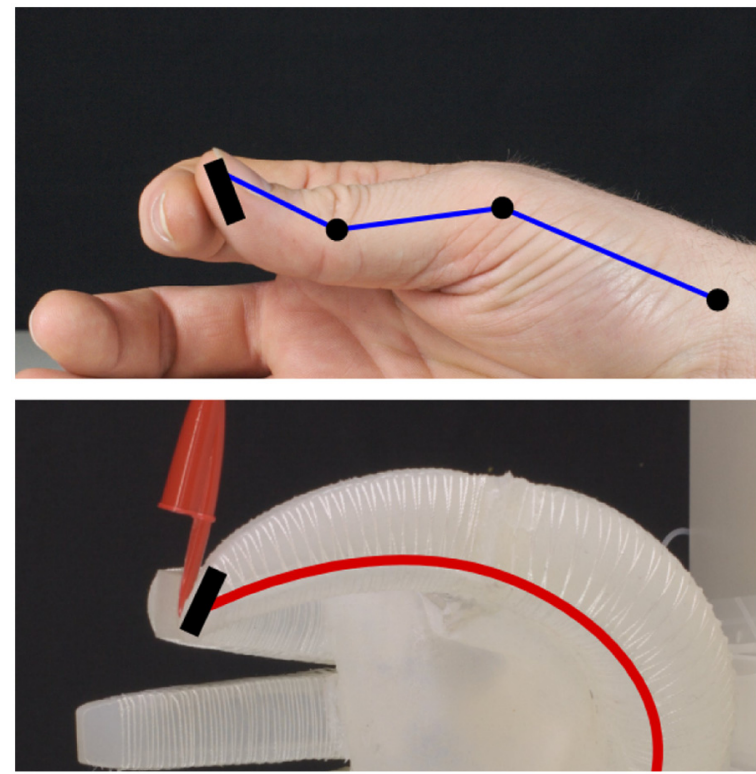

Fig. 6. Difference in thumb configuration and fingertip use during a pincer grasp between a human hand and the robotic hand.

the thumb. We therefore deviated here from the human hand. Instead of the inside of the thumb, we use the backside (dorsal side) as the primary contact surface for pincer grasps. This effectively changes the contact surface orientation by about $45-60^{\circ}$, relative to the orientation found in a human thumb, avoiding the need for negative curvatures. As both sides of the PneuFlex actuator have similar surface characteristics (unlike human thumbs), this choice will not affect grasp quality.

\subsection{Scaffold}

The fingers and the palm are connected to the wrist by individual, flexible struts as part of a three-dimensional printed polyamide scaffold ( $2 \mathrm{~mm}$ thick, see Figure 1$)$. The intentionally flat cross section of the struts enables deformation modes, such as arching the palm and spreading the fingers. Space for the respective actuator is provisioned, but was not added to the hand described here. The struts decouple displacement between fingers, further increasing passive compliance of the hand. The flexibility of the struts limits impact forces, while providing sufficient stiffness for heavy payloads without excessive deformation (see Figure 11).

The fingers and the palmar actuator compound are bonded to the supporting scaffold as shown in Figure 1. The palm is supported by parts of the scaffold to increase its torsional stiffness during opposition with the fingers.

\subsection{Strength between thumb and fingers}

A flexible, but inextensible band connects the base of the index finger to that of the thumb (see Figure 4). Similarly 


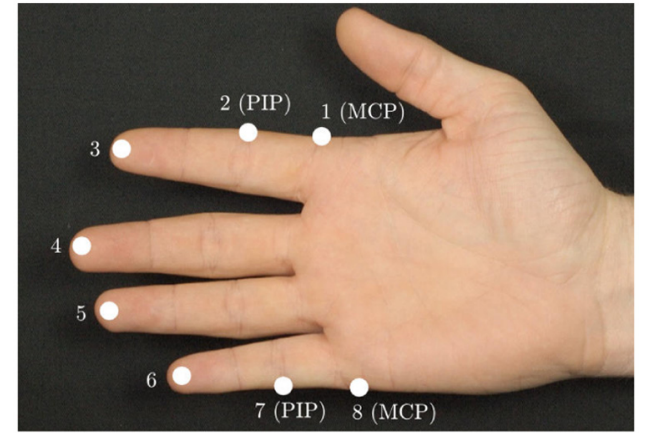

Fig. 7. The Kapandji test counts the number of indicated locations that can be contacted with the thumb tip.

to a muscle in human hands (adductor Pollicis), it enables increased contact forces between thumb and opposing finger, by reducing torques on the struts at the wrist. The sheet connecting fingers and palm serves a similar role, especially for power grasps of cylindrical objects with large diameter.

\section{Grasp dexterity}

In this section, we evaluate the dexterous grasping capabilities of the proposed hand. The most appropriate evaluation would of course be in full-fledged, real-world grasping experiments. However, this requires the integration of hand and control with perception and grasp planning and would effectively be an evaluation of the integrated system. Here, we focus on evaluating the capabilities offered by the hand. Furthermore, we have to resort to empirical methods. Accurate simulation of the complex, nonlinear deformations encountered in such a heterogeneous and soft structure is difficult to conduct and anyways requires empirical experiments to validate the results.

\subsection{Thumb dexterity}

Medical doctors employ the Kapandji test (Kapandji, 1986) to assess thumb dexterity during rehabilitation after injuries or surgery. This test was also used by Grebenstein for evaluating and improving the thumb dexterity of the Awiwi hand (Grebenstein, 2012). For the Kapandji test, the human subject has to touch a set of easily identifiable locations on the fingers with the tip of the thumb. These locations are shown in Figure 7. The total number of reachable locations serves as an indicator of overall thumb dexterity. A thumb is considered fully functional if it is able to reach all locations.

To perform the Kapandji test on our hand, we manually selected actuation pressures that would position the thumb as desired. The postures of the hand performing the test are shown in Figure 8. The thumb tip could reach all but one location. Location 1 was not possible to reach because it would require a backwards bending of actuator 5 (thumb).
Still, the hand scores seven out of eight points, indicating a high thumb dexterity.

\subsection{Grasp postures}

A common way of assessing the dexterous grasping capabilities of hands is to demonstrate grasps for a set of objects. For example, the THE Second Hand was evaluated with 4 objects and 2 grasp types (Grioli et al., 2012), the SDM hand on 10 objects and a single grasp type (Dollar and Howe, 2008), the Velo Gripper on 12 objects and a single grasp type (Ciocarlie et al., 2013), and the Awiwi hand on 8 objects and 16 grasp types (Grebenstein, 2012). We follow these examples in our evaluation.

We select grasp types and objects based on the most comprehensive grasp taxonomy to date, the Feix taxonomy (Feix et al., 2009). It covers the grasps most commonly observed in humans and therefore is a realistic reference for assessing the dexterity necessary for common grasping tasks. The taxonomy encompasses 33 grasp types, out of which the first 17 are identical to the grasps in the Cutkosky taxonomy (Cutkosky, 1989). To demonstrate these 33 grasps, the original publication illustrates 17 different object shapes (Feix et al., 2009). We therefore used 17 objects and 33 grasp types to evaluate our hand.

We implemented the grasps from the Feix taxonomy by defining appropriate actuation pressures and actuation sequences. When, due to collisions, simultaneous actuation of all channels was not sufficient to reach the desired posture, we added an appropriate pre-grasp posture. The commanded actuation pattern was then modified and tested iteratively to improve the quality of the grasp in terms of grasp stability and robustness against external forces, and to ensure the proper types and locations of contact. Grasp quality was judged by manually rotating and translating the hand, and by testing several repetitions of the actuation pattern.

To simplify the search for appropriate actuation patterns, we combined the control of the seven actuators into four actuation channels. Channel A drives actuators 1, 2, and 3 (small, ring, and middle fingers), channel B drives actuator 4 (index finger), channel $\mathrm{C}$ drives actuators 5 (thumb) and 7 (inner palm), and channel D controls actuator 6 (outer palm). These channels can be understood as the hand's four grasping synergies.

To perform a grasping experiment for a particular grasp type, the experimenter triggers the actuation sequence to attain the pre-grasp posture, holds the object in the seemingly most appropriate location relative to the hand, and then triggers the actuation sequence for the grasping motion. The resulting postures for each empirical actuation pattern are shown in Figure 12; high-resolution images are provided in Extension 1. Out of 33 grasp types, the hand is able to perform 31 repeatedly (three consecutive successful trials). The two grasps that failed are the light tool grasp and the distal type grasp. 

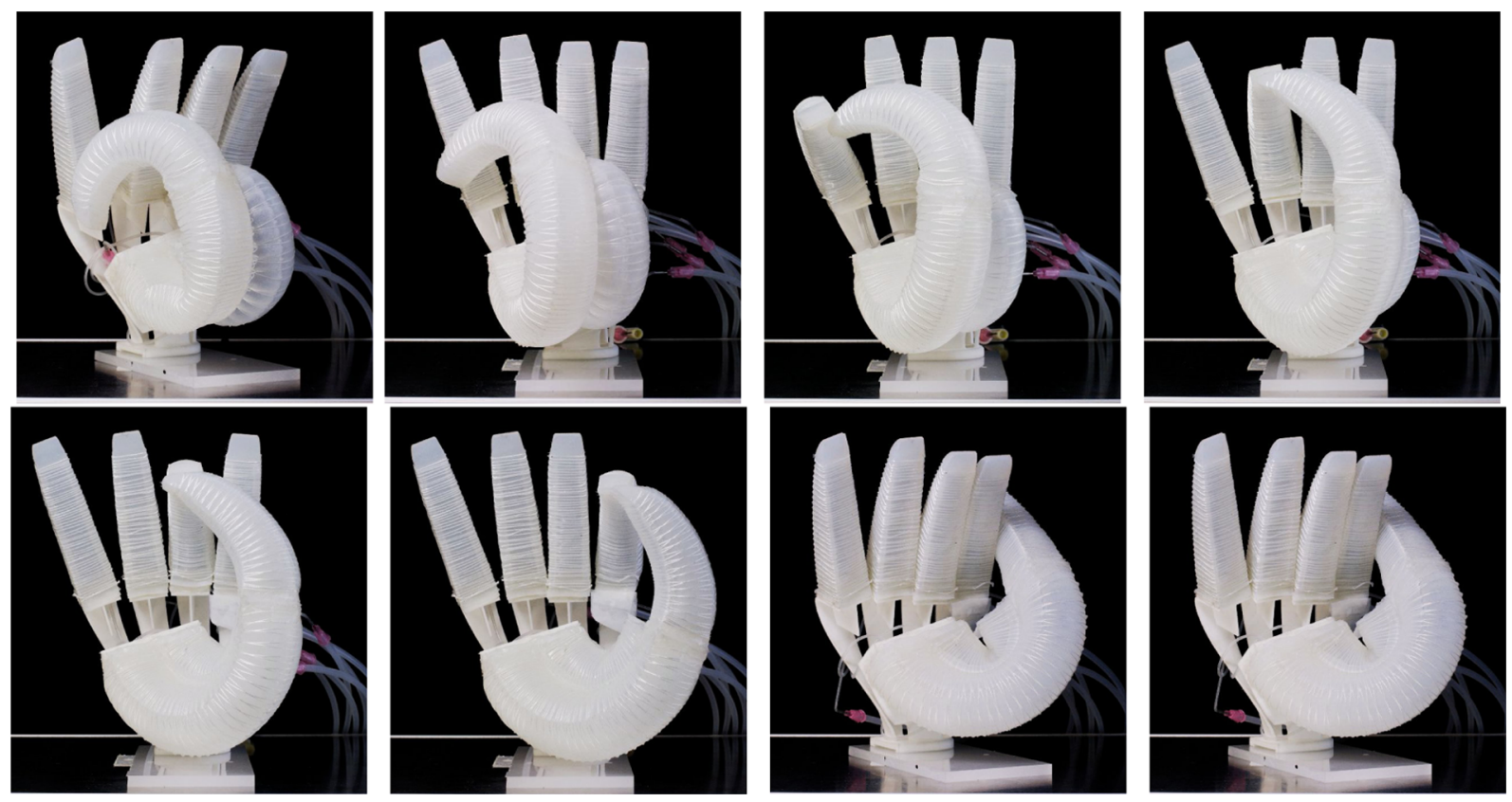

Fig. 8. The RBO Hand 2 succeeds in the Kapandji test for all but one position (position 1, lower right, showing best effort).

The light tool grasp fails because the hand does not possess finger pulp that fills the cavity formed by the maximally bent fingers, which causes the object to slip. The distal type grasp fails because the resulting grasp is nonfunctional with respect to proper use of the scissors, even though it is possible to put the soft fingers through the scissors' holes. Both grasp failures are shown in Figure 9.

Figure 10 shows a scatter plot of the actuation patterns for the 31 successfully achieved grasp types of the Feix taxonomy. The actuation patterns relate to final grasps, not pre-grasp postures. The plots indicate an even distribution of activation for all channels and do not reveal obvious correlations that could be leveraged to further simplify actuation.

The evaluation presented in this section demonstrates the hand's ability to assume a variety of grasp postures. This ability is comparable with that of other hands presented in the literature. We therefore believe that dexterous grasping and compliance can indeed be combined in a highly capable, compliant, underactuated robotic hand.

\subsection{Grasping forces}

While grasp quality and grasp strength was not the driving design criterion for the hand, it is important to verify that a compliant hand is capable of lifting objects of reasonable weight. To give the reader an intuition on the capabilities of the hand, we provide a few tests regarding grasping forces.

The heaviest objects used in the Feix taxonomy grasps were the rectangular plate in grasp $22(156 \mathrm{~g})$, the metal disk in grasp $10(181 \mathrm{~g})$, the wooden ball in grasp 26 $(183 \mathrm{~g})$, and the circular plate in grasp $30(240 \mathrm{~g})$. Note that in grasps 26 and 30, the shown posture offers the least structural support of possible hand poses. Figure 11 shows two additional heavy objects, a wooden cylinder (541 g) and a lead ball $(1.650 \mathrm{~g})$. Figure 11 also shows three different directional disturbance forces on a cylinder which is power-grasped with grasp 1 . If forces above $6-8 \mathrm{~N}$ are applied, the cylinder will slide in the hand.

\subsection{Grasping in realistic settings}

To further illustrate the effectiveness of the proposed hand, we performed experiments with complete grasping sequences, shown in Figure 13 and in Extension 2. In these experiments, a human operator selects the appropriate grasp, triggers the pre-grasp posture of the hand, places the hand in the appropriate location, and then executes the grasp. These experiments demonstrate that the proposed hand, given appropriate perception and grasp planning skills, is able to perform real-world grasps.

\section{Compliance benefits dexterous grasping}

In the previous section, we showed that our underactuated and compliant hand is capable of dexterous grasping. In this section we will investigate whether its compliance and underactuation are beneficial or detrimental to attaining different grasp postures. If beneficial, control will be simpler than the resulting behavior, i.e. actuation space is smaller than grasp posture space. The dimensionality of the posture space that exceeds the dimensionality of the actuation space can be explained by the compliant interactions between hand and object. 

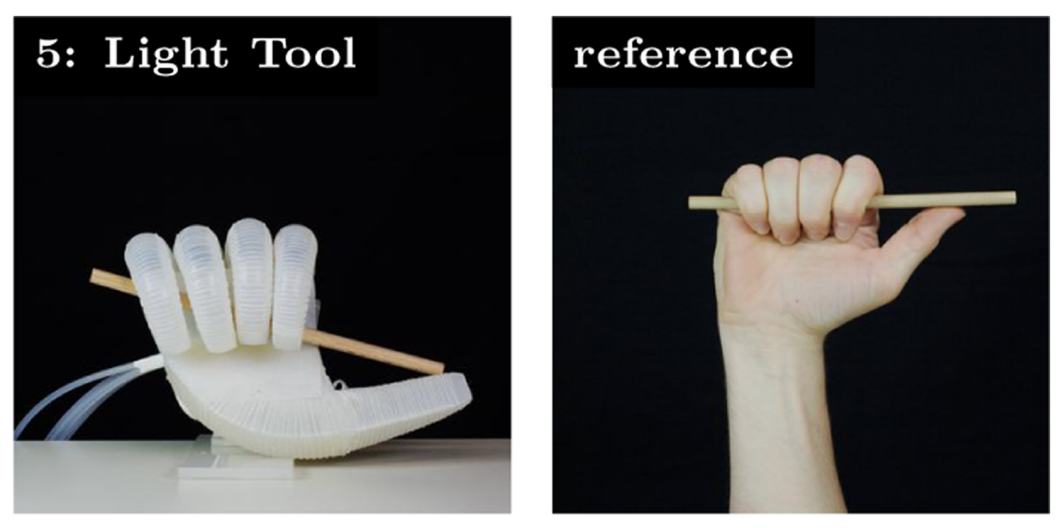

(a) Failed Light Tool grasp: no force closure
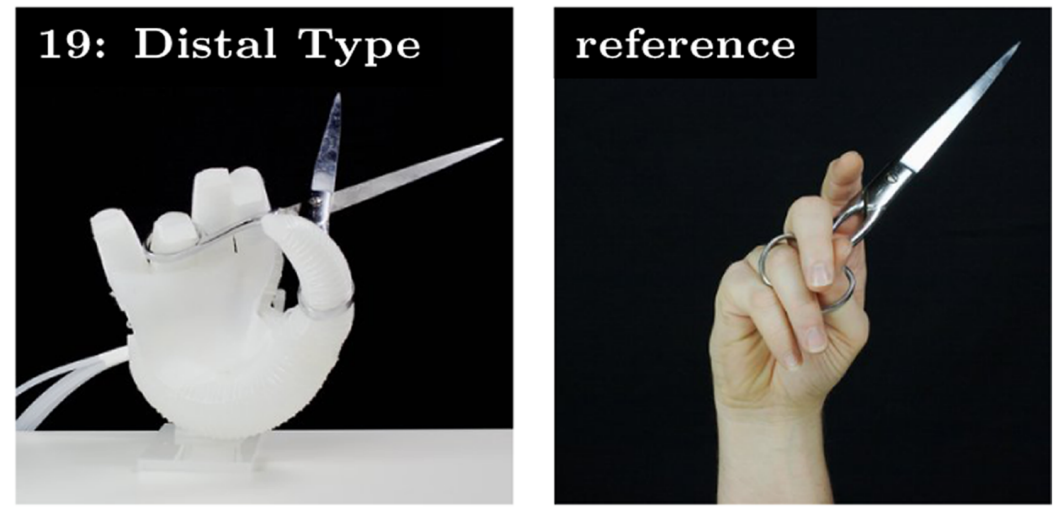

(b) Failed Distal Type grasp: functionally defective

Fig. 9. Grasping postures not successfully attained by the robotic hand.
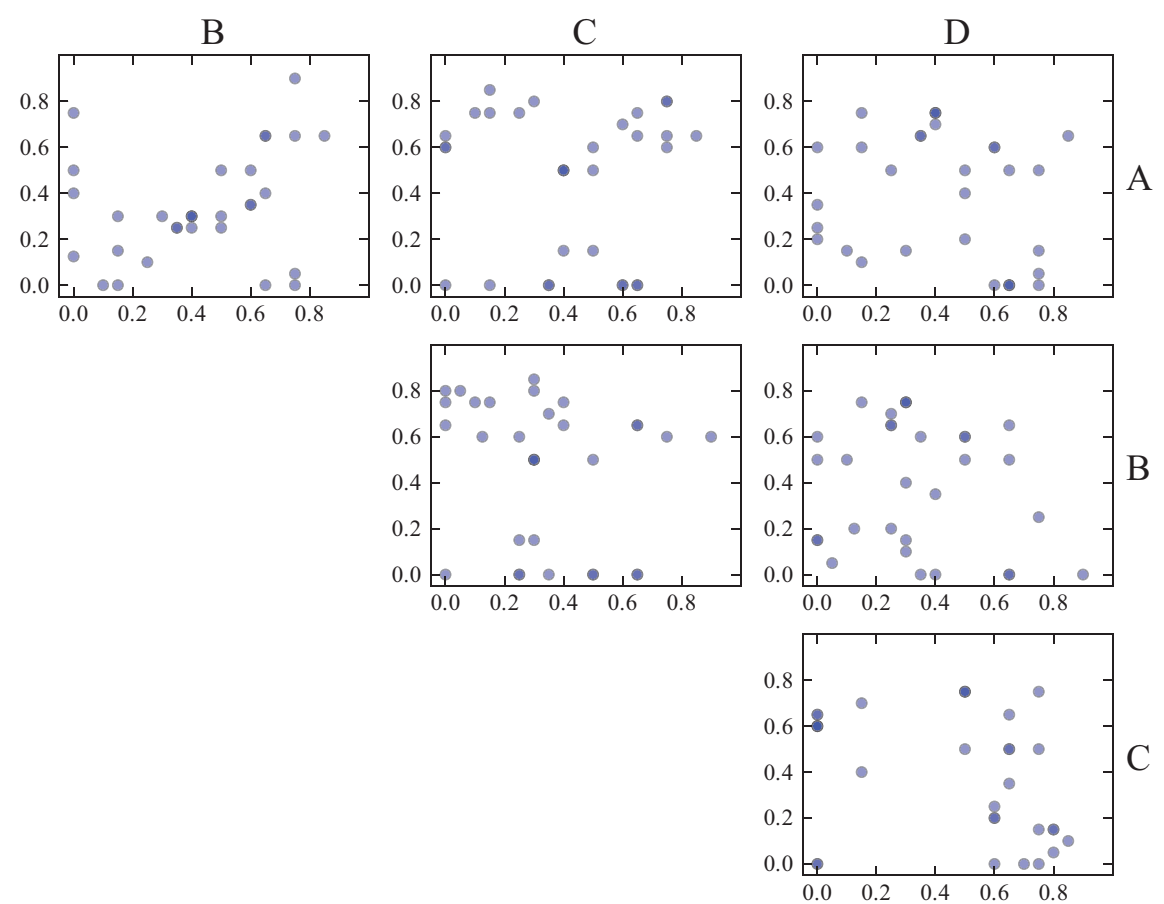

Fig. 10. Scatter plots of the four actuation channels for the actuation patterns of the 31 successful grasps. Darker color indicates overlapping dots. 


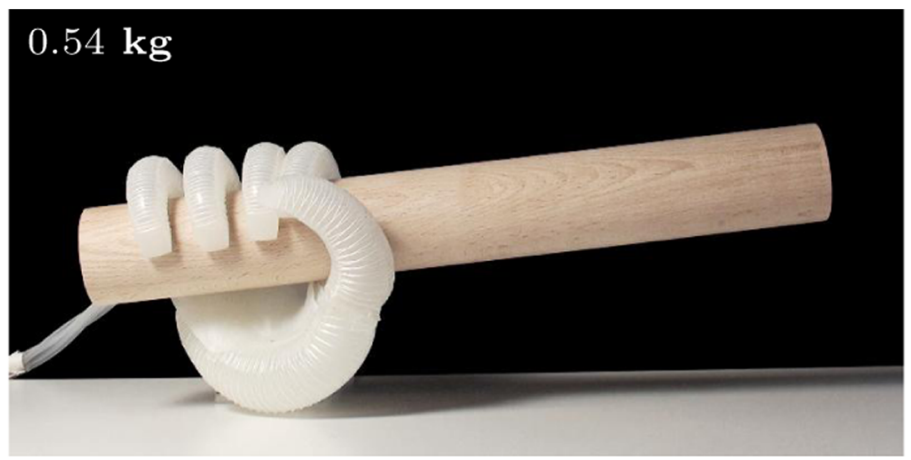

(a) Cylinder off center

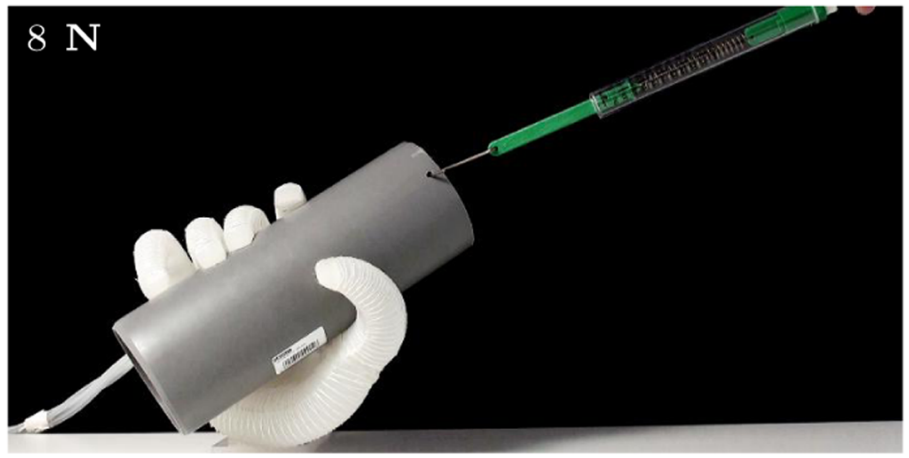

(b) Tolerated disturbance

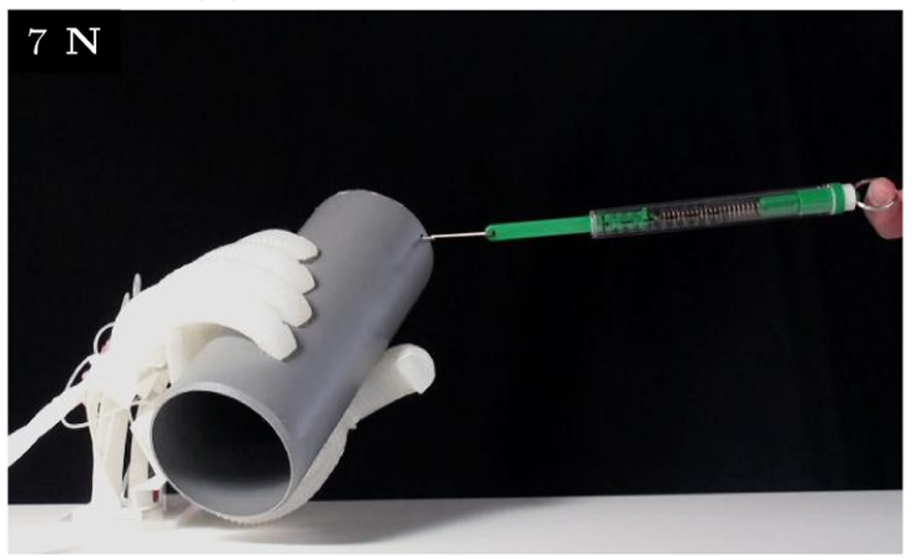

(c) Tolerated disturbance

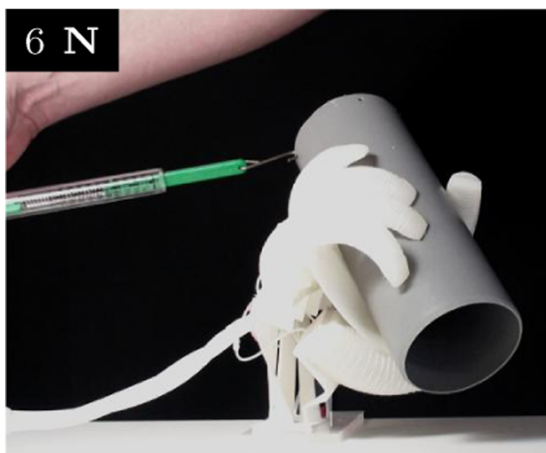

(d) Tolerated disturbance

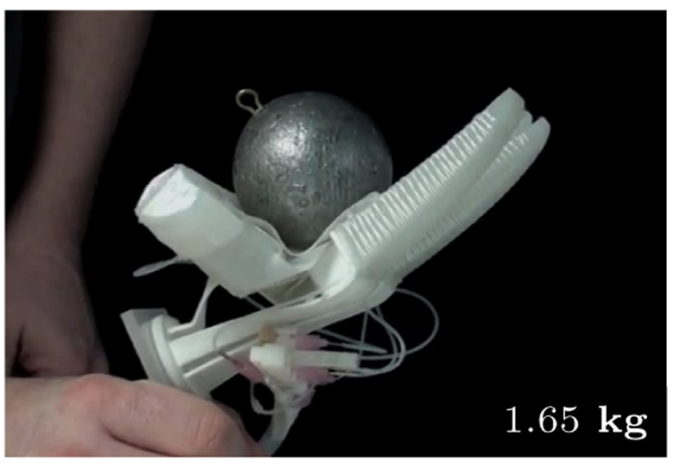

(e) Support strength

Fig. 11. Illustrations of grasping force capabilities: (a) finger strength and palm support strength, (b)-(d) tolerated disturbance forces in different directions for grasp 1, and (e) strength of the support provided by the scaffold. 

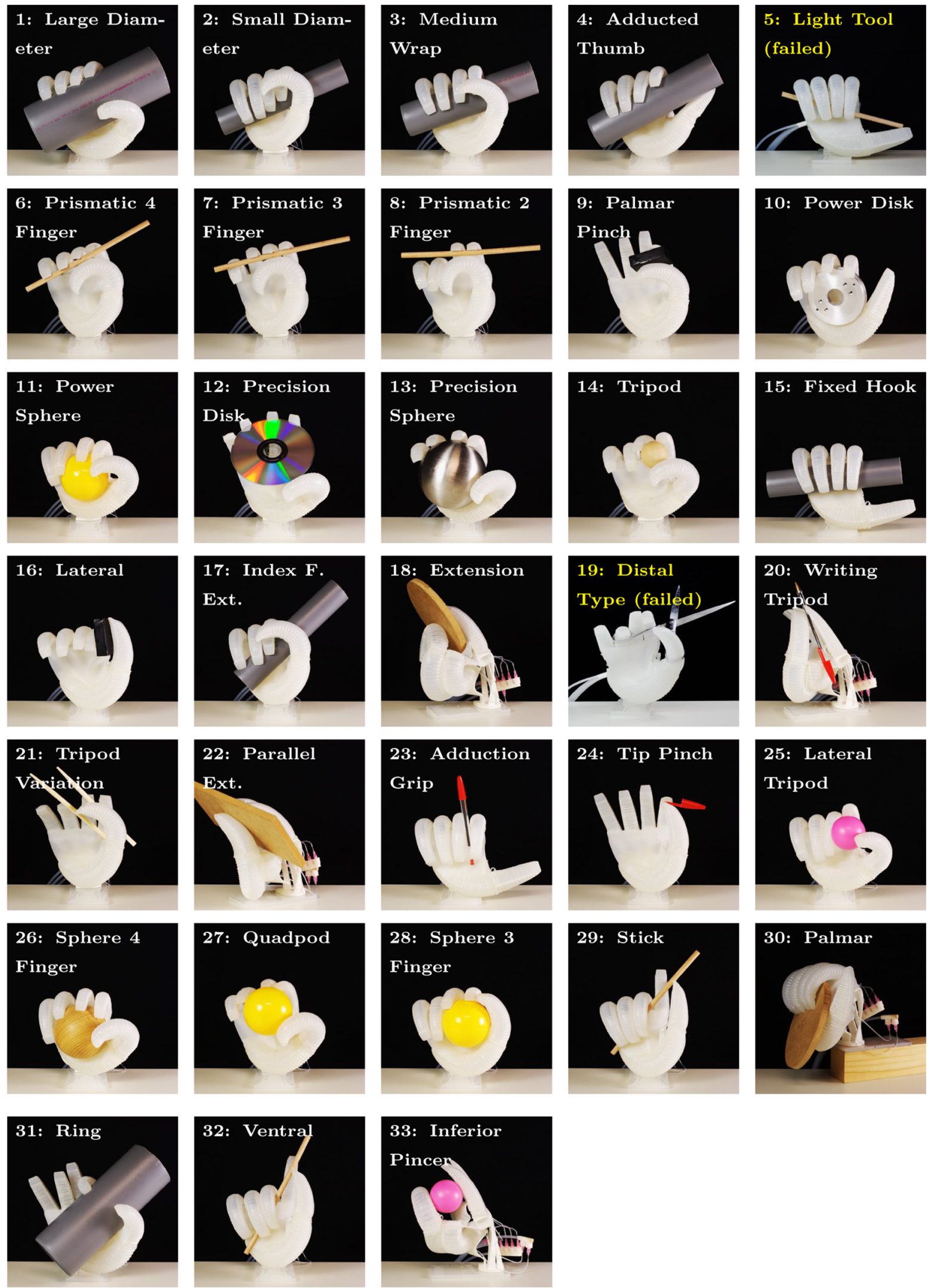

Fig. 12. Enacted grasps of the Feix taxonomy, using empirically determined actuation patterns: Grasps are numbered according to the Feix taxonomy (Feix et al., 2009); the hand failed to replicate grasps 5 (Light Tool) and 19 (Distal Type, Scissors). 

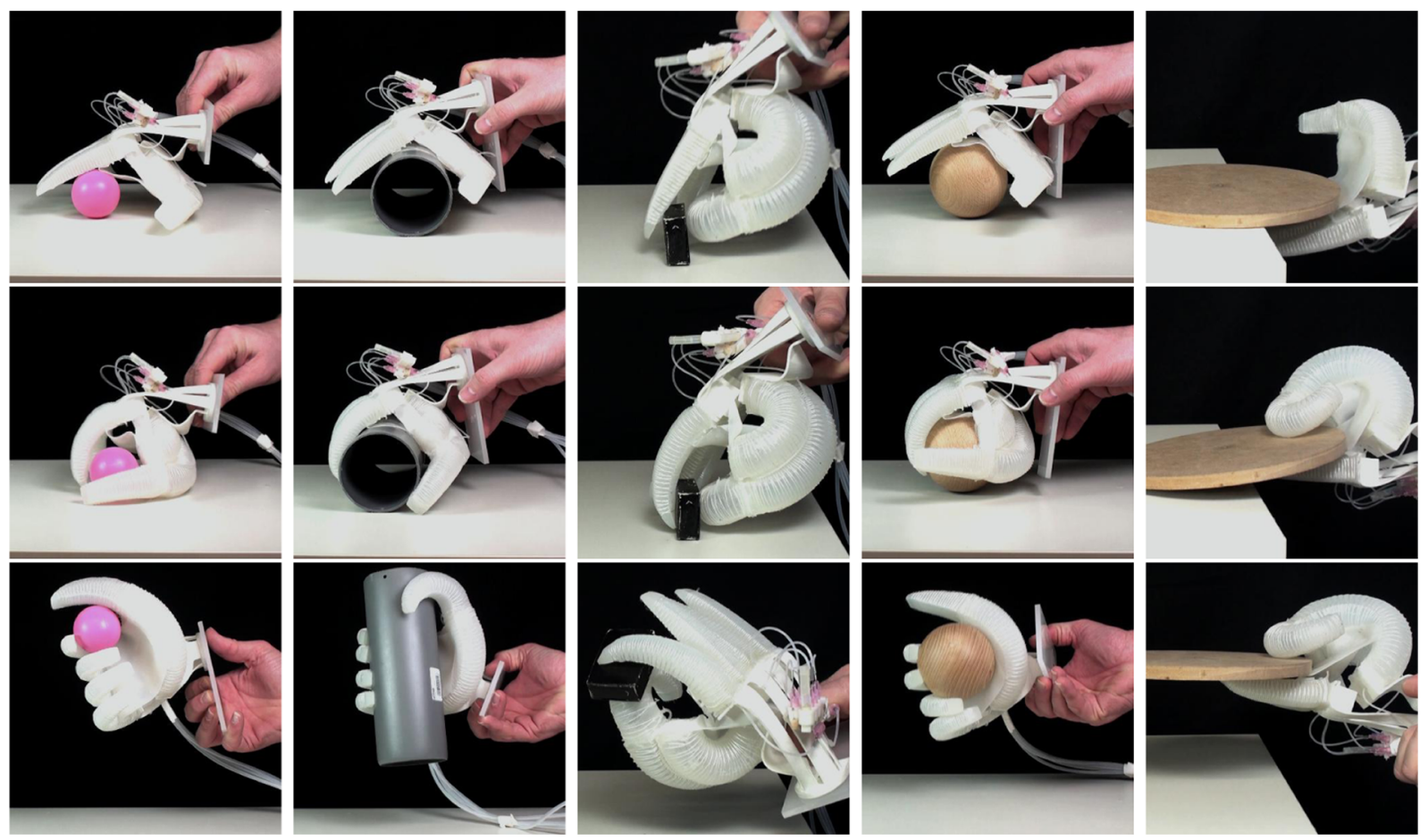

Fig. 13. Performing grasps using the grasp postures 25, 1, 9, 28 and 18: a human places the hand and then triggers the actuation of the appropriate grasp; top, pre-grasp posture; middle, executed grasp; bottom, lifting object to show success.

\subsection{Postural diversity of the Feix taxonomy}

To assess the dimensionality of the attainable grasp posture space, we first have to assert that the grasp set we use to sample from that space is diverse enough, i.e. that the employed grasps span the space of possible grasps. For this, we recorded humans doing Feix taxonomy grasps using the method published by Santello et al. (1998), and compare the results with existing published data sets (the data published in Santello et al. (1998), the UNIPI data set, ${ }^{1}$, and UNIPI-ASU data set.

In the experiment, we asked five healthy human participants to enact every grasp of the Feix taxonomy five times while wearing a Cyberglove II data glove, using exactly the same objects as used for the experiment in the previous section. Participants were allowed to use the other hand to assist in assuming the grasp posture, but had to achieve a successful grasp in the sensorized hand without additional support. The resulting postures were sampled 50 times within $500 \mathrm{~ms}$ and averaged over samples and episodes. We then performed dimensionality reduction by applying principal component analysis (PCA) for each subject individually to exclude inter-subject variance in accordance with the data analysis used in Santello et al. (1998). We then compared the resulting residual unexplained variances with data from literature.

The results are shown in Figure 14. For four out of five participants, the unexplained variances were higher than those of the three independently published data sets, suggesting that the grasps span the space of possible grasp

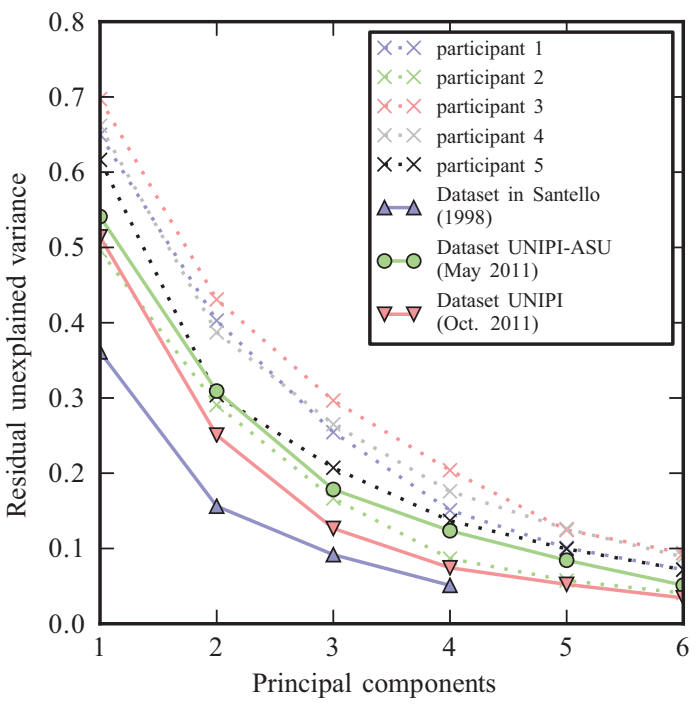

Fig. 14. Residual variances of PCA on joint measurements taken with data gloves. Solid lines denote published data sets using the method of Santello et al. (1998), dotted lines denote the data acquired using the Feix taxonomy and the objects shown in Figure 12.

postures more effectively than the considerably larger but less structured set of objects that was used for the data sets we compare with.

The discrepancy between the published data sets and ours may be explained by the fact that the Santello and UNIPI data sets were recorded on grasping imagined 


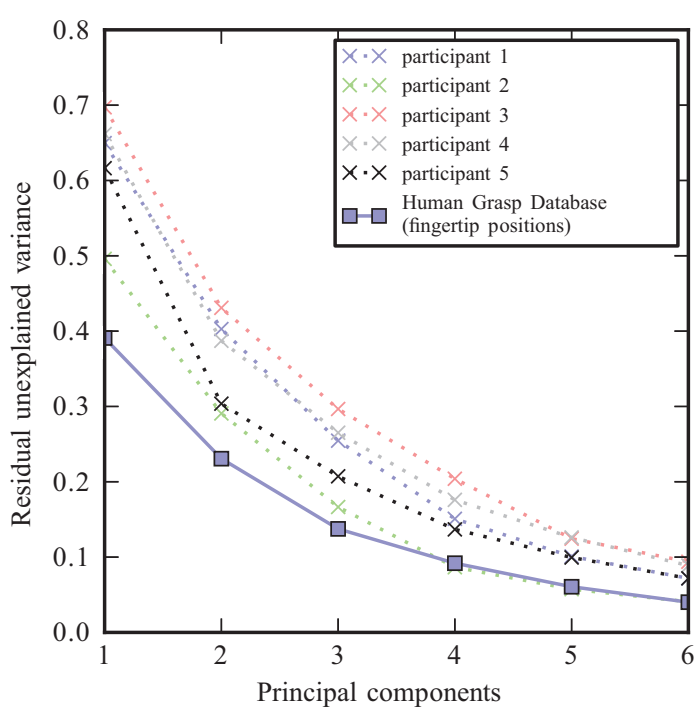

Fig. 15. Residual variances of PCA on grasp postures enacted by humans using data from different acquisition methods, joint angles and fingertip positions. Solid line denotes data from the Human Grasp Database (Romero et al., 2010), dotted lines denote the data acquired using the Feix taxonomy and the objects shown in Figure 12.

objects, while our data and the UNIPI-ASU data set are recorded while grasping real objects. The additional variance observed may come from the interaction between hand and object, whereas with imagined objects, hand posture may be more related to actuation pattern than the actual grasp posture. This interpretation of existing data is consistent with our hypothesis that hand control can be simpler than effected posture.

\subsection{Choice of representation}

Because the RBO Hand 2 does not have discrete joints, it is not possible to assess its postural diversity using the method of Santello et al. (1998), even though the hand is anthropomorphic. An alternative representation of hand posture compatible with soft hand mechanics has been used for the Human Grasp Database experiment. In this experiment, Romero et al. (2010) represented hand posture in terms of fingertip position and orientation relative to the back of the hand.

Before using this representation, we first have to establish that for assessing postural diversity it is comparable to using joint angles. Figure 15 shows a comparison of the residual unexplained variances of a PCA for fingertip position and joint angles (from the previous experiment), both acquired on human hands using the Feix taxonomy (31 grasps, excluding grasps 19 and 23 in Figure 12). The graph shows that fingertip positions may be a more compact representation than joint angles, but most importantly not worse. Therefore, we can use fingertip position data as a conservative estimate of joint posture diversity. Omitting fingertip orientation data (represented as quaternions) from

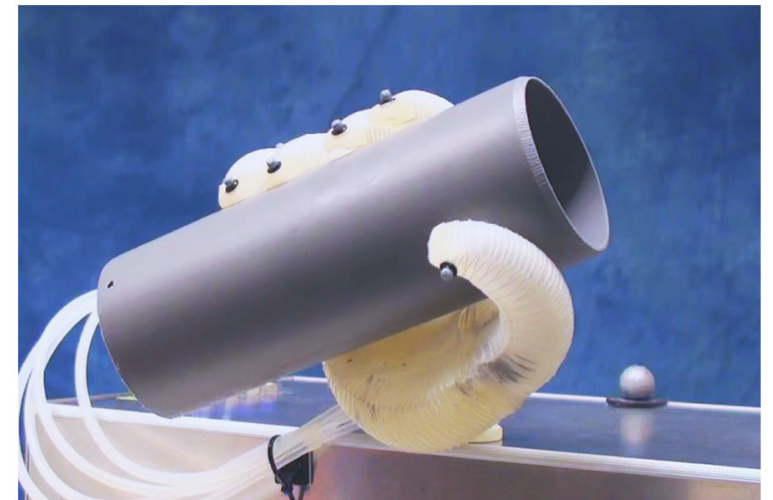

Fig. 16. Screen shot from video Extension 4, showing the marker placement on the fingertips for Motion Capture.

the PCA did not significantly decrease unexplained variance. It was therefore excluded to simplify acquisition of comparable data on the RBO Hand 2 using a motion capture system.

Concluding the human experiments and comparative study of published data, the Feix taxonomy appears to be a good proxy for diverse grasp postures. In addition, we found that fingertip position is a viable alternative to joint angles for assessing postural diversity of human hands and therefore also of anthropomorphic robot hands.

\subsection{Postural diversity of RBO Hand 2}

The previous subsection gives us a justification to use fingertip positions of grasps from the Feix taxonomy to assess the RBO Hand 2 in terms of grasp posture diversity. To acquire the fingertip positions we used a motion capture system. We placed $3 \mathrm{~mm}$ retroreflective markers on the backside of the fingertips of the four fingers. On the thumb tip, the marker was placed on the side to not interfere with the pincer grasp. The marker placement can be seen in Figure 16 as in the video Extension 4. Note that additional markers were attached to the hand during recording, but only the five fingertip markers were used for the experiment.

The motion capture system has a spatial precision of $0.05 \mathrm{~mm}$, as measured using 205 frames recorded for a stationary hand (at $50 \mathrm{~Hz}$ ). For each grasp, two trials are recorded, fingertip positions are extracted and averaged over the trials before applying PCA. The data set from the Human Grasp Database is subjected to the same procedure. The results are shown in Figure 17. The postural dimensionality exhibited by the RBO Hand 2 matches the human hand data well. This strongly supports our claim that our robotic hand is as dexterous as a human hand with respect to attainable grasp posture, taking the Feix taxonomy as a reference.

A second observation can be made by considering the dimensionality of control required to implement the grasp postures, which is indicated by the dotted line and gray 


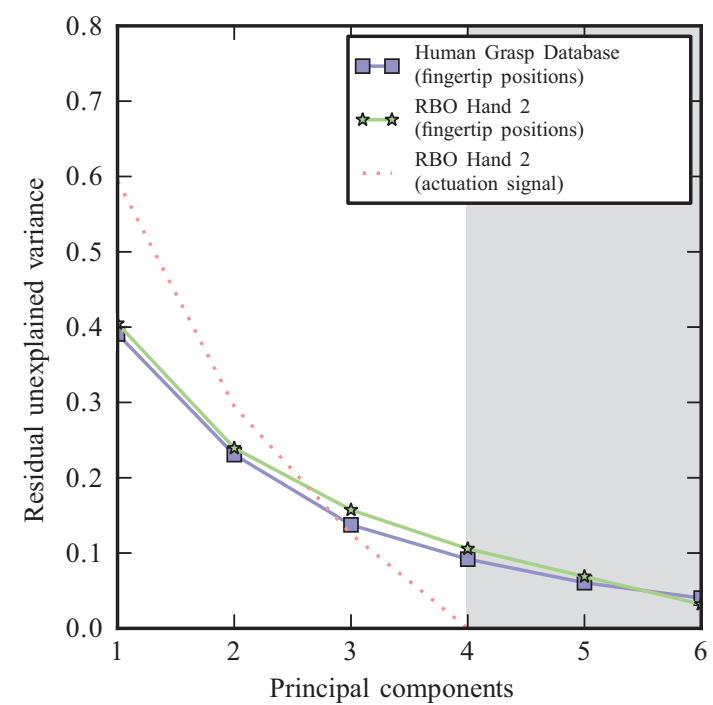

Fig. 17. Dimensionality of fingertip positions for a human hand, the RBO Hand 2, and its respective control signal. The gray region indicates where the dimensionality of fingertip posture exceeds control dimensionality.

area in Figure 17. The space of grasp postures is of higher dimensionality than the actuation space, which is of dimension four. Where does this increase come from? It must be introduced by the diverse shapes of the grasped objects. The interactions between the hand and the object differentiate different postures. This differentiation is facilitated by the hand's ability to adapt to objects compliantly. The differences between imagined and real grasps in human experiments, discussed in Section 6.1, corroborate this hypothesis. Tavakoli et al. (2014) also obtain the minimum of four to six actuated degrees of freedom to implement dexterous grasping for their anthropomorphic, compliant hand.

The diversity and consistency of evidence we presented here strongly suggests that compliant hands benefit dexterous grasping.

The presented evaluation, based on published data sets, measurements of human grasps, and of grasps with the RBO Hand 2, paints a consistent picture: the robotic hand performs similar to a human hand, both in terms of grasp posture diversity, and in terms of covering sets of grasps, again, using the Feix taxonomy as a reference.

This diversity in grasping posture is achieved by the RBO Hand 2 with only four actuated degrees of freedom. This is possible because the interaction of the compliant degrees of freedom with the diverse objects introduces additional variance in the posture.

\section{Limitations}

Adopting a novel technology in a new application, like continuum actuators in the design of soft dexterous hands, opens up new possibilities but also leads to new limitations and challenges that need to be considered carefully.

\subsection{Grasp forces and payload}

Continuum actuators, when constructed with reinforced rubber and actuated hydraulically, are in principle capable of exerting extremely large forces. For example, an actuator made out of car tire rubber with steel-fiber reinforcements and hydraulic actuation would probably be able to exert grasping forces exceeding $100 \mathrm{~N}$. It would also be straightforward to make a much stronger hand with the current production process by choosing stiffer rubbers and thicker hulls. In the current hand design, we chose to use very soft actuators, to investigate the effect of compliance, to increase safety, and to make manufacturing convenient. We chose pneumatic actuation over other fluidic options as it is much simpler and cleaner to operate in a lab environment.

\subsection{Grasp stiffness}

While grasp forces, as discussed above, refer to the magnitude of forces exerted on the object, grasp stiffness refers to the hand's ability to maintain a grasp posture in the presence of external forces. Naturally, a soft hand built for maximum compliance does not create an extraordinarily stiff grasp. Low grasp stiffness has the advantage of reducing the peak forces encountered at the contact points between object and hand. It also reduces the probability of slip when objects collide or upon jerky wrist motion. At the same time, it also places a limit on the forces the hand can exert on the environment through the grasped object, for example. This negatively affects tasks where those forces must be high. We therefore need to balance and these two competing properties.

In case grasp stiffness proves to be the limiting factor for certain tasks, there are several methods available to selectively increase actuator stiffness (Wall et al., 2015). But they increase design complexity and production costs, and therefore should be avoided if possible. Another simple method to increase grasp stiffness is to select power grasps instead of precision grasps.

In the context of grasp stiffness, we can understand the exploration of soft hand designs as searching for a lower bound on grasp stiffness that still is able to provide sufficient grasp force. Future research will investigate how grasp stiffness can be increased while maintaining compliance where necessary.

\subsection{Pneumatics}

Our hand relies on additional external pneumatic components for control. These components are cheap and readily available in industry-grade quality. However, they are oversized for the low pressures, small volumetric flow, and size constraints of robotic hands. Miniaturizing and integrating electrically actuated valves directly into the hand, possibly even into the actuator, would greatly simplify integration into predominantly electromechanic robots. 
Long-term autonomy arguably is easier to achieve with pneumatic systems. In contrast to electrical power systems, where no good solution for long-term untethered operation exists, the technology exists to make small, quickly refillable air tanks or even to directly convert chemical energy (Wehner et al., 2014).

Mobility can easily be obtained by using compact, small compressors, as the average rate of airflow for operating the RBO Hand 2 is very low and peaks can be serviced by small air tanks. The use of electrical energy storage also often simplifies the integration into existing system.

\subsection{Precision and repeatability of grasps}

While actuation patterns can be reproduced with high precision, the interaction with the object or features of the environment during a grasp can introduce substantial variations in the final grasp posture. While this is often understood to be a disadvantage, we view it as an important feature of the design, leading to robust grasping performance. Therefore, we believe one must carefully differentiate between precision and repeatability versus grasp robustness. The hand presented in this paper deliberately trades the former for the latter.

\subsection{Sensing}

The compliance of the materials makes local, dense sensing for proprioception and contact forces important but also very difficult. Also, sensor technologies compatible with soft actuators are currently not available commercially. While it is easy to integrate air pressure sensors, it would be very desirable to integrate strain and touch sensors too. This is a topic of active research as current electronic sensor technology is predominantly designed for rigid structures, but working solutions for stretchable electronics start to appear, making an integration with PneuFlex actuators feasible in the near future (Culha et al., 2014; Gerratt et al., 2014; Rahimi et al., 2014).

\subsection{Modeling hand mechanics}

Modeling the whole hand poses certain challenges: an accurate mechanical state is difficult to obtain due to nonlinearities arising from large deformation and anisotropic structure of its components. In addition, the actuators intentionally provide a large number of deformation modes, which increases hand complexity even further and makes sensing the hand's complete mechanical state very difficult.

Because of these features, we are currently not able to provide a quantitative analysis of grasp quality based on mechanical models as it is state of the art for hands with rigid links. We therefore qualitatively assess grasp quality in Section 5.3 and also provide two videos in Multimedia Extensions 5 and 6 that illustrate the attainable quality of grasps.
To start closing the gap in modeling soft continuum actuators, we present a model for computing key mechanical properties of PneuFlex actuators in Appendix B. While that model is not able to estimate all parameters necessary to create a faithful simulation, it can be used to straightforwardly customize deformation modes and stiffness profiles of the actuators before building them. Appendix B also evaluates the influence of several nonlinear phenomena on actuator behavior and suggests several, easy to follow design guidelines to avoid common failure modes.

Yet another consequence of the complex and highly variable deformations that happen during grasping is that most existing grasp planners cannot be applied, as they rely on complete and accurate geometric and kinematic models of hand and environment. While it might be possible to perform simulations of the hand's deformation using finite element methods, these are computationally too complex to employ them in search-based grasp planning.

\section{Conclusions}

We have presented a compliant, underactuated, and dexterous anthropomorphic robotic hand based on soft robotics technology. The hand is able to achieve 31 of 33 grasp postures from a state-of-the-art human grasp taxonomy. To evaluate the dexterity of the opposable thumb, we performed the Kapandji test, in which the hand achieves seven out of eight possible points. We illustrated the hand's excellent payload to weight ratio, as it is able to lift objects of nearly three times its own weight. We also presented realworld grasping experiments to demonstrate the hand's capabilities in a realistic setting.

We believe that compliance is crucial to enable robust grasping in robotic hands. We provided support for this statement by showing that the dimensionality of the achievable postural space is significantly larger than the dimensionality of the hand's actuation space. We explain this observation with the hand's ability to mechanically comply to the shape of the grasped object: The final grasping posture is the result of the hand's actuation together with compliant interactions between the hand and the object. We found that several grasping experiments with humans are consistent with this interpretation. We therefore conclude that compliance in robotic hands, when used correctly, can facilitate not only robustness in power grasps but also dexterity.

In addition to enhancing dexterity, the use of soft robotic technology renders the hand robust to impact and blunt collisions and makes it inherently safe and suitable for working environments containing dirt, dust, or liquids. The effort, complexity, and cost of building the hand are significantly lower than for existing hand technologies. The hand presented here can be built in 2 days, using materials worth less than US\$100. Both actuator and hand structure are easily adaptable to specific application domains. We therefore believe that this novel way of building robotic hands 
significantly lowers the barrier to entry in the field of grasping and manipulation research.

\section{Funding}

This work was supported by the Alexander von Humboldt foundation through an Alexander von Humboldt professorship (funded by the German Federal Ministry of Education and Research), the SOMA project (European Commission, grant number H2020-ICT645599) and the German Research Foundation (DFG, award number BR 2248/3-1).

\section{Notes}

1. Data set available at http://handcorpus.org

2. Data set available at http://handcorpus.org

3. Data set available at http://grasp.xief.net/

\section{References}

Amend J, Brown E, Rodenberg N, Jaeger H and Lipson H (2012) A positive pressure universal gripper based on the jamming of granular material. IEEE Transactions on Robotics 28(2): 341-350.

Bae J, Park S, Park J, Baeg M, Kim D and Oh S (2012) Development of a low cost anthropomorphic robot hand with high capability. In: IEEE/RSJ international conference on intelligent robots and systems (IROS), pp. 4776-4782.

Bishop-Moser J, Krishnan G, Kim C and Kota S (2012) Design of soft robotic actuators using fluid-filled fiber-reinforced elastomeric enclosures in parallel combinations. In: IEEE/RSJ international conference on intelligent robots and systems (IROS), pp. 4264-4269.

Catalano MG, Grioli G, Farnioli E, Serio A, Piazza C and Bicchi A (2014) Adaptive synergies for the design and control of the pisa/IIT SoftHand. The International Journal of Robotics Research 33(5): 768-782.

Ciocarlie M, Mier Hicks F and Stanford S (2013) Kinetic and dimensional optimization for a tendon-driven gripper. In: IEEE international conference on robotics and automation (ICRA), pp. 2751-2758.

Controzzi M, Cipriani C and Carozza MC (2014) Design of artificial hands: A review. In: The Human Hand as an Inspiration for Robot Hand Development (Springer Tracts in Advanced Robotics, vol. 95). New York: Springer, pp. 219-247.

Culha U, Nurzaman SG, Clemens F and Iida F (2014) SVAS3: Strain vector aided sensorization of soft structures. Sensors 14(7): $12748-12770$.

Cutkosky MR (1989) On grasp choice, grasp models, and the design of hands for manufacturing tasks. IEEE Transactions on Robotics and Automation 5(3): 269-279.

Deimel R and Brock O (2013) A compliant hand based on a novel pneumatic actuator. In: IEEE international conference on robotics and automation (ICRA), pp. 2047-2053.

Deimel R and Brock O (2014) A novel type of compliant, underactuated robotic hand for dexterous grasping. In: Proceedings of robotics: science and systems, Berkeley, CA.

Deimel R, Eppner C, Alvarez-Ruiz J, Maertens M and Brock O (2013) Exploitation of environmental constraints in human and robotic grasping. In: 16th international symposium on robotics research (ISRR).
Dollar AM and Howe RD (2008) Simple, reliable robotic grasping for human environments. In: IEEE international conference on technologies for practical robot applications (TePRA), pp. 156-161.

Dollar AM and Howe RD (2010) The highly adaptive SDM hand: Design and performance evaluation. The International Journal of Robotics Research 29(5): 585-597.

Feix T, Pawlik R, Schmiedmayer H, Romero J and Kragic D (2009) A comprehensive grasp taxonomy. In: Robotics, science and systems: workshop on understanding the human hand for advancing robotic manipulation.

Gabiccini M, Farnioli E and Bicchi A (2013) Grasp analysis tools for synergistic underactuated robotic hands. The International Journal of Robotics Research 32(13): 1553-1576.

Gaiser I, Schulz S, Kargov A, et al. (2008) A new anthropomorphic robotic hand. In: 8th IEEE-RAS international conference on humanoid robots (Humanoids), pp. 418-422.

Galloway KC, Polygerinos P, Walsh CJ and Wood RJ (2013) Mechanically programmable bend radius for fiber-reinforced soft actuators. In: 16th international conference on advanced robotics (ICAR), pp. 1-6.

Gent AN (2012) Engineering with rubber: how to design rubber components. Carl Hanser Verlag GmbH Co KG.

Gerratt A, Sommer N, Lacour S and Billard A (2014) Stretchable capacitive tactile skin on humanoid robot fingers; first experiments and results. In: 2014 14th IEEE-RAS international conference on humanoid robots (Humanoids), pp. 238-245.

Giannaccini ME, Georgilas I, Horsfield I, et al. (2014) A variable compliance, soft gripper. Autonomous Robots 36(1-2): 93-107.

Giorelli M, Renda F, Arienti A, et al. (2012) Inverse and direct model of a continuum manipulator inspired by the octopus arm. In: Biomimetic and Biohybrid Systems (Lecture Notes in Computer Science, number 7375). New York: Springer, pp. 347-348.

Grebenstein M (2012) Approaching Human Performance - The Functionality Driven Awiwi Robot Hand. Dissertation, ETH Zürich, Zürich.

Grioli G, Catalano M, Silvestro E, Tono S and Bicchi A (2012) Adaptive synergies: an approach to the design of underactuated robotic hands. In: IEEE/RSJ international conference on intelligent robots and systems (IROS), pp. 1251-1256.

Hirose S and Umetani Y (1978) The development of soft gripper for the versatile robot hand. Mechanism and Machine Theory 13(3): 351-359.

Ilievski F, Mazzeo A, Shepherd RF, Chen X and Whitesides GM (2011) Soft robotics for chemists. Angewandte Chemie International Edition 50(8): 1890-1895.

Kapandji IA (1986) Cotation clinique de l'opposition et de la contre-opposition du pouce. Annales de Chirurgie de la Main 5(1): 68-73.

Kazemi M, Valois J, Bagnell JA and Pollard N (2014) Humaninspired force compliant grasping primitives. Autonomous Robots 37(2): 209-225.

Ma R, Odhner L and Dollar A (2013) A modular, open-source 3D printed underactuated hand. In: IEEE international conference on robotics and automation (ICRA), pp. 2737-2743.

Marchese A, Komorowski K, Onal C and Rus D (2014) Design and control of a soft and continuously deformable $2 \mathrm{D}$ robotic manipulation system. In: IEEE international conference on robotics and automation (ICRA), pp. 2189-2196.

Meier P, Lang M and Oberthür S (2005) Reiterated tension testing of silicone elastomer. Plastics, Rubber and Composites 34(8): 372-377. 
Mosadegh B, Polygerinos P, Keplinger C, et al. (2014) Pneumatic networks for soft robotics that actuate rapidly. Advanced Functional Materials 24(15): 2163-2170.

Odhner L, Jentoft L, Claffee M, et al. (2014) A compliant, underactuated hand for robust manipulation. The International Journal of Robotics Research 33(5): 736-752.

Prattichizzo D, Malvezzi M, Gabiccini M and Bicchi A (2012) On the manipulability ellipsoids of underactuated robotic hands with compliance. Robotics and Autonomous Systems 60(3): 337-346.

Rahimi R, Ochoa M, Yu W and Ziaie B (2014) A sewing-enabled stitch-and-transfer method for robust, ultra-stretchable, conductive interconnects. Journal of Micromechanics and Microengineering 24(9): 095018.

Renda F, Cianchetti M, Giorelli M, Arienti A and Laschi C (2012) A 3D steady-state model of a tendon-driven continuum soft manipulator inspired by the octopus arm. Bioinspiration and Biomimetics 7(2): 025006.

Romero J, Feix T, Kjellström H and Kragic D (2010) Spatio-temporal modeling of grasping actions. In: 2010 IEEE/RSJ international conference on intelligent robots and systems (IROS), pp. 2103-2108.

Santello M, Flanders M and Soechting JF (1998) Postural hand synergies for tool use. The Journal of Neuroscience 18(23): 10105-10115.

Tavakoli $\mathrm{M}$ and Almeida Ad (2014) Adaptive under-actuated anthropomorphic hand: ISR-SoftHand. In: IEEE/RSJ international conference on intelligent robots and systems (IROS), pp. 1629-1634.

Tavakoli M, Enes B, Marques L and Almeida AD (2014) Actuation strategies for underactuated anthropomorphic hands. In: IEEE/RSJ international conference on intelligent robots and systems (IROS), pp. 274-280.

Wall V, Deimel R and Brock O (2015) Selective stiffening of soft actuators based on jamming. In: IEEE international conference on robotics and automation (ICRA). Available at: http:// www.robotics.tu-berlin.de/fileadmin/fg170/Publikationen_pdf/ ICRA15_0055_FI.pdf.

Wehner M, Tolley MT, Mengüç, et al. (2014) Pneumatic energy sources for autonomous and wearable soft robotics. Soft Robotics 1(4): 263-247.

\section{Appendix A: Index to Multimedia Extensions}

Archives of IJRR multimedia extensions published prior to 2014 can be found at http://www.ijrr.org, after 2014 all videos are available on the IJRR YouTube channel at http:// www.youtube.com/user/ijrrmultimedia

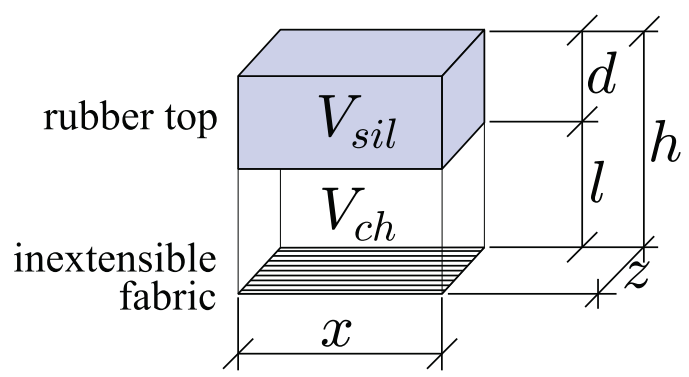

Fig. 18. Simple model of an actuator segment.

\section{Appendix B: Derivation of an approximate PneuFlex model}

To facilitate the design of PneuFlex actuators, we present a computational model for its deformation. Overall, the deformation of such an actuator is complex. Nevertheless, surprisingly simple design rules can be derived for designing the most important actuator properties, namely, actuation ratio and rotational stiffness around the actuated axis. This appendix proposes a suitable formalization, an analysis of simplifications taken, and a basic experimental evaluation of the resulting equations.

\section{B.1 Formalization of the PneuFlex actuator geometry}

Figure 18 shows the parameterization of a small segment of the actuator. To simplify the model, we ignore the side walls and assume a rectangular cross-section. Let $x, z, d$ be the length, width, and thickness of a segment of the actuator, respectively.

Width and height of the actuator are assumed to not change due to the helical thread around the actuator. This assumption is facilitated by selecting an approximately circular cross-section (e.g. a square) for the shape of actuator cross-section, as the radial fibers will always deform the shape into a circle to balance the radial pressure.

Due to its embedded fabric, the bottom layer also has a fixed length $x$. The only possible deformation left is to

Table of Multimedia Extensions

\begin{tabular}{lll}
\hline Extension & Media type & Description \\
\hline 1 & Images & High resolution images of grasps from the Feix taxonomy in Figure 12 \\
2 & Data & Fingertip positions of RBO Hand 2 for the Feix taxonomy \\
3 & Data & Joint angles of five human participants executing grasps from the Feix taxonomy recorded with a \\
& & Cyberglove II \\
4 & Video & Video of how grasp postures for grasps from the Feix taxonomy were obtained \\
6 & Video & Video of example grasps from a tabletop under human control \\
6 & Video & Video of manipulating two heavy objects \\
\hline
\end{tabular}




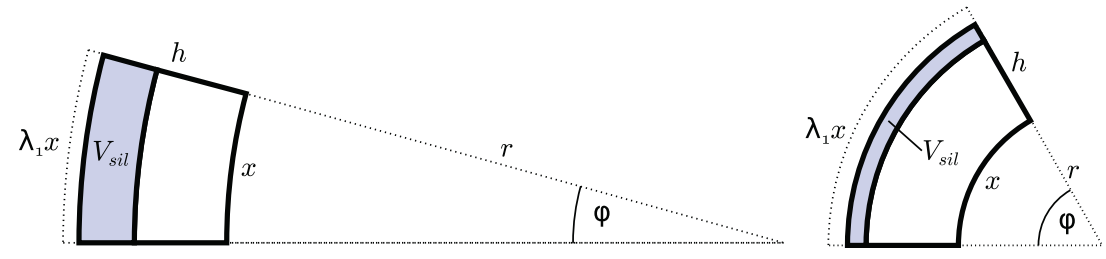

Fig. 19. Parameterization of an actuator segment, illustrated at different curvatures. Here $x, V_{\text {sil }}$ and $h$ stay constant while $\lambda, r$ and $\varphi$ change.

stretch the silicone layer while bending the bottom layer, which is illustrated in Figure 19

The interfaces of the segment are rotated to each other by the angle $\varphi$ and the bottom layer curves with the radius $r=\frac{x}{\varphi}$. As we also have configurations with $\varphi=0$ (when the actuator is deflated), we will rather express equations in terms of the curvature $\kappa$ of the bottom layer:

$$
\kappa=\frac{1}{r}=\frac{\varphi}{x} \Rightarrow \varphi=\kappa \cdot x
$$

We can also express the curvature on the top side of the actuator:

$$
\kappa_{\text {top }}=\frac{1}{\frac{1}{\kappa}+h}
$$

Both $\kappa$ and $\kappa_{\text {top }}$ are defined on the same angle $\varphi$. Therefore, we can compute a relationship between angle $\varphi$ and stretch $\lambda_{1}$ :

$$
\begin{gathered}
\varphi=\varphi_{\text {top }} \\
\kappa \cdot x=\frac{1}{\frac{1}{\kappa}+h} \cdot \lambda_{1} x \\
\kappa=\frac{\lambda_{1}-1}{h} \\
\lambda_{1}=1+\kappa \cdot h \\
\varphi=\frac{x}{h} \cdot\left(\lambda_{1}-1\right)
\end{gathered}
$$

Volumes. To aid readability of the analysis, we define several constant volumes that do not change under deformation. These volumes can be computed from the basic actuator geometry:

The volume of the effectively incompressible silicone in the active layer of the actuator:

$$
V_{s i l}=z \cdot x \cdot d
$$

The volume of the deflated air chamber:

$$
V_{c h}=z \cdot x \cdot(h-d)
$$

The total volume of the deflated actuator:

$$
\begin{aligned}
V_{a c t} & =z \cdot x \cdot h=\frac{h}{d} \cdot V_{\text {sil }} \\
& =V_{c h}+V_{\text {sil }}
\end{aligned}
$$

The total volume of air contained in the deflated actuator plus any volumes connected to it, such as supply tubes:

$$
V_{\text {air }}=V_{\text {supply }+V c h}=V_{\text {supply }}+V_{\text {act }}-V_{\text {sil }}
$$

The symbol $V$ will denote the actual volume of the air chamber, which is dependent on the deformation. Therefore it is a function of actuator curvature. The total actual volume of the actuator is $V+V_{\text {sil }}$.

Volume change. For computing the energy stored by the compressed gas (air) within the actuator, we need to compute the actual volume with respect to actuator curvatures. We can do this by first calculating the total volume of a flexed actuator segment:

$$
\begin{aligned}
V+V_{\text {sil }} & =z \cdot \frac{\varphi}{2 \pi} \cdot\left(\pi \cdot\left(\frac{x}{\varphi}+h\right)^{2}-\pi \cdot\left(\frac{x}{\varphi}\right)^{2}\right) \\
& =V_{a c t} \cdot\left(1+\frac{h}{2} \frac{\varphi}{x}\right)
\end{aligned}
$$

As $\frac{\varphi}{x}=\kappa$, we can express actuator curvature in terms of air chamber volume and initial geometry as

$$
\begin{aligned}
V+V_{\text {sil }} & =V_{a c t} \cdot\left(1+\frac{h}{2} \cdot \kappa\right) \\
\kappa & =\frac{2}{h}\left(\frac{V+V_{\text {sil }}}{V_{a c t}}-1\right) \\
& =\frac{2}{h} \cdot\left(\frac{V-V_{c h}}{V_{a c t}}\right)
\end{aligned}
$$

With Equation (1) we can also compute the relationship between $\lambda_{1}$ and the actuator volume:

$$
\lambda_{1}=1+2 \cdot\left(\frac{V-V_{c h}}{V_{a c t}}\right)
$$

This leads to the first insight: actuator curvature and stretch of the silicone rubber are linearly proportional to the gas volume: 


$$
\begin{aligned}
\partial \kappa & =\frac{2}{V_{a c t} \cdot h} \cdot \partial V \\
\partial \lambda_{1} & =\frac{2}{V_{a c t}} \cdot \partial V
\end{aligned}
$$

Strain tensor invariants. The energy stored in the rubber during deformation is modeled using strain tensor invariants (Gent, 2012). The strain tensor invariants are related to the orthogonal stretches $\lambda_{1}, \lambda_{2}, \lambda_{3}$ :

$$
\begin{aligned}
& J_{1}=\lambda_{1}^{2}+\lambda_{2}^{2}+\lambda_{3}^{2}-3 \\
& J_{2}=\lambda_{1}^{2} \lambda_{2}^{2}+\lambda_{1}^{2} \lambda_{3}^{2}+\lambda_{2}^{2} \lambda_{3}^{2}-3 \\
& J_{3}=\lambda_{1} \cdot \lambda_{2} \cdot \lambda_{3}-1
\end{aligned}
$$

To compute them, we first define actuator-specific relations between stretches $\lambda_{1}, \lambda_{2}$ and $\lambda_{3}$ in three principal directions, which are aligned to $x, h$ and $z$, respectively.

Silicones are effectively incompressible, therefore we can assume a constant volume:

$$
\lambda_{1} \cdot \lambda_{2} \cdot \lambda_{3}=1
$$

The actuator's radial size does not change either because of the reinforcement helices. We therefore set the circumferential stretch $\lambda_{3}=1$, and obtain the relationship

$$
\lambda_{2}=\lambda_{1}^{-1}
$$

This deformation is also called pure shear. Using these relations, both $J_{1}$ and $J_{2}$ reduce to

$$
J_{2}=J_{1}=\lambda_{1}^{2}+\lambda_{1}^{-2}-2
$$

and because of the incompressibility assumption:

$$
J_{3}=0
$$

Simplifications and limitations. To keep the model simple, many potentially important effects were not included. We assume a uniform strain energy density within the rubber hull, which is acceptable for moderately thin rubber hulls (i.e. $d<0.5 h$ ). This assumption is modeled and discussed in Section B.6.

We use a neo-Hookean material model. This ignores higher-order deformation effects. The error is less than 3.4\% though, as discussed in Section B.7.

We ignore material stiffening. The consequences are discussed in Section B.8. The resulting error is typically less than $7.7 \%$.

Finally, we also ignore the side walls, i.e. hull parts of the actuator which are stretched only at fractions of $\lambda_{1}$. The ramifications are discussed in Section B.9.

The model can also be invalidated by compressive forces applied externally. They remove fiber tension and therefore usually lead to buckling. This limits the usefulness of the model for simulation and planning. The main purpose is to provide simple equations for designing actuator behavior though. Here the limitations are acceptable.

\section{B.2 Statically stable actuator configurations}

Using the minimum potential energy principle we can derive statically stable configurations for the actuator First, we need to define all relevant forms of work in the system:

- gas compression;

- elastic rubber deformation;

- work added by an external load.

The total potential energy of the actuator is

$$
W=W_{\text {air }}+W_{\text {sil }}+W_{\text {load }}
$$

Equilibrium is reached, when the gradient of work is zero, which is in our case very simple as we will describe the actuator state with the single variable $\lambda_{1}$ :

$$
\frac{\Delta W}{\Delta \lambda_{1}}=0
$$

The remainder of this section derives each work component from the definitions of the previous section and yields the equation for statically stable configurations.

Gas compression work. For computing the gas compression work, we need to differentiate between two different control regimes.

In the mathematically simpler case, pressure is held constant under deformation: $p(V)=p$. This is either done actively by using a control system or passively by using a big reservoir connected to the actuator volume, which attenuates the effect of volume change within the actuator on pressure.

In the second, more common case the total enclosed gas mass in the system is held constant e.g. when using pneumatic valves. Pressure changes according to the ideal gas equation: $p(V)=n R T \cdot \frac{1}{V}$. A closed gas volume increases the stiffness of the actuator slightly. But for the sake of brevity, the latter case will not be derived here.

In both cases, the work done by changing the volume of a gas from $V_{1}$ to $V_{2}$ is

$$
W_{\text {air }}=W_{V_{1}}-\int_{V_{1}}^{V_{2}} p(V) \cdot d V
$$

In the case of constant gas pressure, we obtain a simple equation:

$$
\begin{aligned}
W_{\text {air }} & =W_{0}-p \cdot(V) \\
\frac{\delta W_{\text {air }}}{\delta \lambda_{1}} & =-p \cdot \frac{\delta V}{\delta \lambda_{1}} \\
\frac{\delta W_{\text {air }}}{\delta \lambda_{1}} & =-p \cdot \frac{1}{2} V_{\text {act }}
\end{aligned}
$$

Rubber deformation work. The deformation work of the silicone rubber $W_{\text {sil }}$ is modeled as a neo-Hookean solid model with coefficient $C_{10}=\frac{G}{2}$ : 


$$
W_{s i l}=\int_{V_{s i l}} \frac{G}{2} \cdot J_{1} \cdot \delta V
$$

$G$ is the material's shear modulus, and $V_{\text {sil }}$ the volume of the silicone. Choosing this simple model over more complex ones is discussed in Section B.7. We will further assume a uniform deformation, and thus uniform strain energy density (i.e. uniform $J_{1}$ ) throughout the hull, as justified in Section B.6. We can then calculate the total strain energy as

$$
W_{\text {sil }}=\frac{G}{2} \cdot J_{1} \cdot V_{\text {sil }}
$$

Using Equation (5), we can express the work gradient with respect to stretch $\lambda_{1}$ :

$$
\begin{aligned}
W_{\text {sil }} & =\frac{G}{2} \cdot\left(\lambda_{1}^{2}+\lambda_{1}^{-2}-2\right) \cdot V_{\text {sil }} \\
\frac{\delta W_{\text {sil }}}{\delta \lambda_{1}} & =G \cdot\left(\lambda_{1}-\lambda_{1}^{-3}\right) \cdot V_{\text {sil }}
\end{aligned}
$$

Load work. For a given actuator segment, external load is applied on the interfaces to the two adjacent segments. By attaching our frame of reference to one interface, load work can be computed by only considering the motion and force of the other interface.

The load work can further be split up into the work done by translatory forces and rotary moments. Translatory forces are transmitted by the inelastic fibers of reinforcement helix and passive layer. If we assume completely inelastic fibers, those forces do not contribute any work. Rotations, on the other hand, do contribute work, and we can integrate the contribution along the bottom layer:

$$
W_{\text {load }}=\int M(\varphi) \cdot d \varphi
$$

For the model, it is more convenient to integrate over $x$ instead of $\varphi$ along the actuator segment. We can rewrite the integral to

$$
W_{\text {load }}=\int M(\varphi(x)) \cdot \frac{\partial \varphi(x)}{\partial x} d x
$$

For short enough actuator segments (small $x$ ) we can assume constant, averaged moment along the whole segment, i.e. $M(x)=M$. In addition we can substitute the derivative of Equation (3) for $\frac{\partial \varphi(x)}{\partial x}$ :

$$
\begin{aligned}
W_{\text {load }} & =\int M \cdot \frac{\lambda_{1}-1}{h} \cdot d x \\
& =M \cdot \frac{\lambda_{1}-1}{h} \cdot x+W_{0}
\end{aligned}
$$

From this equation, we can compute the work gradient with respect to stretch $\lambda_{1}$ :

$$
\frac{\delta W_{\text {load }}}{\delta \lambda_{1}}=M \cdot \frac{x}{h}
$$

Minimum total potential energy. By computing the local minimum of Equation (6) with respect to $\lambda_{1}$ and substituting with Equations (7), (8) and (9), we obtain the equation describing stable actuator states:

$$
\begin{array}{r}
0=\frac{\delta W_{\text {air }}}{\delta \lambda_{1}}+\frac{\delta W_{\text {sil }}}{\delta \lambda_{1}}+\frac{\delta W_{\text {load }}}{\delta \lambda_{1}} \\
0=-p \cdot \frac{V_{\text {act }}}{2}+G \cdot\left(\lambda_{1}-\lambda_{1}^{-3}\right) V_{\text {sil }}+M_{\text {load }} \cdot \frac{x}{h}
\end{array}
$$

\section{B.3 Stiffness}

The Stiffness of an actuator segment is expressed by the change in moment $M_{\text {load }}$ with respect to curvature $\kappa$. From Equation (10) we can compute $M_{\text {load }}$ it explicitly:

$$
M_{\text {load }}=\frac{h^{2} \cdot z}{2} \cdot p-\left(\lambda_{1}-\lambda_{1}^{-3}\right) \cdot G \cdot h \cdot d \cdot z
$$

As we assume a constant pressure regime, the first term vanishes when differentiating:

$$
\frac{\delta M_{\text {load }}}{\delta \lambda_{1}}=-G \cdot h \cdot d \cdot z \cdot\left(1+3 \cdot \lambda_{1}^{-4}\right)
$$

We then substitute with the derivative of Equation 2 which is $\partial \lambda_{1}=h \cdot \partial \kappa$. In addition, we can set $z=h$, as the width of the actuator is usually approximately its height. We arrive at the equation describing the stiffness of the actuator given its shape and deformation:

$$
\frac{\delta M_{\text {load }}}{\delta \kappa}=h^{3} \cdot G \cdot d \cdot\left(1+3 \lambda_{1}^{-4}\right)
$$

The last, nonlinear term predicts a strong stiffening when a PneuFlex actuator is straight or even negatively curved. This sudden stiffening is indeed observed with actual actuators. But they also tend to buckle under such loads.

Scaling law. When scaling an actuator, the ratios $\frac{d}{h}$ and $\frac{z}{h}$ stay constant. The equation then becomes:

$$
\frac{\delta M_{\text {load }}}{\delta \kappa}=h^{4} \cdot\left(G \cdot \frac{d}{h} \cdot\left(1+3 \lambda_{1}^{-4}\right)\right)
$$

Stiffness therefore scales to the fourth power of actuator size. This gives us a powerful lever to adjust the strength of an actuator.

Non-squared cross-sections. Because the stable configuration of the helical thread from the applied uniform radial pressure is a circle, the cross-section will always deform into one given high enough air pressure.

It therefore makes sense to use the actuator circumference $c$ instead of height and width to compute actuator stiffness:

$$
\frac{\delta M_{\text {load }}}{\delta \kappa}=\left(\frac{c}{4}\right)^{3} \cdot(G \cdot d) \cdot\left(1+3 \lambda_{1}^{-4}\right)
$$


For designing actuator stiffness, we can approximate the nonlinear term with 1 :

$$
\frac{\delta M_{\text {load }}}{\delta \kappa}=\left(\frac{c}{4}\right)^{3} \cdot(G \cdot d)
$$

\section{B.4 Actuation ratio}

The actuation ratio $\frac{\delta \kappa}{\delta p}$ is the change of curvature given an increase in pressure while assuming zero load. It can also be computed from Equation (10):

$$
\begin{aligned}
p & =\left(\lambda_{1}-\lambda_{1}^{-3}\right) \cdot G \cdot \frac{d}{h} \\
\frac{\delta \lambda_{1}}{\delta p} & =\frac{1}{\left(1+3 \lambda_{1}^{-4}\right) \cdot G \cdot \frac{d}{h}}
\end{aligned}
$$

Using Equation (2) we can substitute $\lambda_{1}$ and $\delta \lambda_{1}$. We obtain the actuation ratio

$$
\frac{\delta \kappa}{\delta p}=\frac{1}{1+3(1+h \kappa)^{-4}} \cdot \frac{1}{G \cdot d}
$$

So according to the simple model, the actuation ratio is inversely proportional to the silicone's shear modulus, and its thickness. The actuation ratio also has a nonlinear component with respect to the actual curvature. At small curvatures the actuation ratio is considerably lower than at higher curvatures. The nonlinearity can be linearized though by a non-circular cross-section, as discussed in Section B.5. For actuator design we can conveniently drop the nonlinear term and arrive at a simple design rule for computing the inverse actuation ratio:

$$
\frac{\delta \kappa}{\delta p} \approx \frac{1}{G \cdot d}
$$

Note that this equation is independent of both $h$ and $z$ (and, therefore, also circumference $c$ ). The actuation ratio is not dependent on the size of the actuator cross-section! We can therefore set an actuation ratio profile using thickness and shear modulus of the rubber hull, and then set the stiffness profile with

$$
\frac{\delta M_{\text {load }}}{\delta \kappa}=\left(\frac{c}{4}\right)^{3} \cdot \frac{1}{\frac{\delta \kappa}{\delta p}} \cdot\left(1+3 \lambda_{1}^{-4}\right)
$$

\section{B.5 Justifying simplification: Linear actuation ratio}

Equation (12) contains the nonlinear term $\left(\lambda_{1}-\lambda_{1}^{-3}\right)$. The model therefore predicts pressure to be nonlinearly related to actuator curvature. Interestingly though, the actuation ratio can be linear at moderate pressures, as was observed by Deimel and Brock (2013). We believe that this is caused by a non-circular cross-section.

When pressure increases, the helical thread tensions and always deforms the actuator cross-section into a circle. But until then, the actuator expands in three dimensions instead

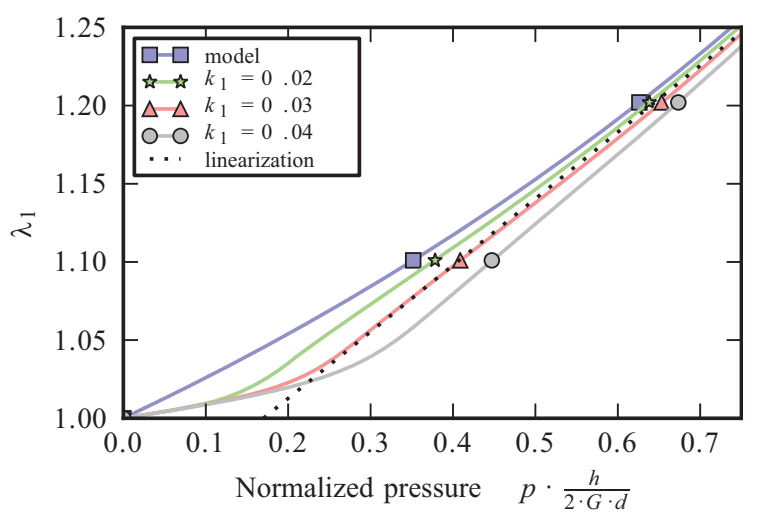

Fig. 20. A non-circular cross-section effectively linearizes the actuation ratio at moderate curvatures.

of one, which increases the strain energy in the rubber hull faster with respect to stretch $\lambda_{1}$. The effect is more pronounced with less circular cross-sections, but can also be elicited by a loosely wound helical thread. We can investigate the resulting effects by introducing a correction factor for the strain energy gradient:

$$
D\left(\lambda_{1}\right)=\left(1-k_{0}\right) \cdot \frac{\left(\lambda_{1}-1\right)^{2}}{\left(\lambda_{1}-1\right)^{2}+k_{1}^{2}}+k_{0}
$$

The factor $k_{0} \approx[1 \ldots 5.0]$ defines the relative increase of the gradient for a deflated actuator, while the factor $k_{1} \approx 0.025$ determines at which elongation the effect is halved. The latter is probably dependent on the wall's thickness and rubber stiffness. The equation was chosen because unlike simpler models the correction factor has a limited range, a finite integral, and no poles.

Then $D\left(\lambda_{1}\right)$ is plugged into Equation (12):

$$
p=D\left(\lambda_{1}\right) \cdot\left(\lambda_{1}-\lambda_{1}^{-3}\right) \cdot 2 \cdot G \cdot \frac{d}{h}
$$

The impact of different $k_{0}$ on actuation ratio is illustrated in Figure 20. The actuator can behave almost linearly at higher pressures. An interesting application of this effect may be to simplify actuator control.

\section{B.6 Justifying simplification: Uniform strain energies within rubber hull}

In the model we assume a uniform strain energy density throughout the hull. This needs to be checked though, as the principal stretches are not uniform at all.

Formalization. For an infinitesimal volume of elastomer within the hull, we define $d$ to be the radial distance of the volume from the outer boundary of the hull in an undeformed actuator, while $d^{\prime}$ denotes the actual radial distance in the deformed actuator.

When the actuator bends, the volume moves radially outwards as the wall thins. This motion influences the 
stretches encountered. As the radial displacement is limited by the helical reinforcement fibers, we can assume the outer boundary of the rubber hull to not move radially at all.

Due to the smaller radius the longitudinal stretch is

$$
\lambda_{1}=1+\left(\kappa \cdot h-\kappa \cdot d^{\prime}\right)
$$

The circumferential stretch can be calculated by the radial displacement given a position $d^{\prime}$ with respect to the undeformed position $d$ :

$$
\begin{aligned}
\delta \lambda_{3} & =\frac{\pi\left(h-d^{\prime}\right)}{\pi(h-d)}-\frac{\pi\left(h-d^{\prime}-\delta d^{\prime}\right)}{\pi(h-d)} \\
& =-\frac{\delta d^{\prime}}{h-d} \\
\Rightarrow \lambda_{3} & =\frac{-d^{\prime}}{h-d}+C
\end{aligned}
$$

Setting the boundary condition $\lambda_{3}=1$ at the outer boundary of the hull yields

$$
\lambda_{3}=\frac{h-d^{\prime}}{h-d}
$$

Finally, we can derive $\lambda_{2}$ by using the incompressibility assumption $\lambda_{1} \lambda_{2} \lambda_{3}=1$ and get

$$
\lambda_{2}=\frac{h-d}{\kappa \cdot h^{2}+\kappa \cdot d^{\prime 2}-(2 \cdot \kappa \cdot h+1) d^{\prime}+h}
$$

To normalize our calculations, we can express the principal stretches in terms of the dimensionless ratios $\frac{d^{\prime}}{h}, \frac{d}{h}$, and $\kappa \cdot h$ :

$$
\begin{aligned}
& \lambda_{1}=1+\kappa \cdot h \cdot\left(1-\frac{d^{\prime}}{h}\right) \\
& \lambda_{2}=\frac{1-\frac{d}{h}}{\kappa \cdot h+\kappa \cdot h \cdot\left(\frac{d^{\prime}}{h}\right)^{2}-(2 \cdot \kappa \cdot h+1) \frac{d^{\prime}}{h}+1} \\
& \lambda_{3}=\frac{1-\frac{d^{\prime}}{h}}{1-\frac{d}{h}}
\end{aligned}
$$

With $\lambda_{2}$ we can express the relation between $d^{\prime}$ and $d$ as a linear differential equation using $\lambda_{2}$ as the gradient of displacement:

$$
\begin{aligned}
& \frac{\delta d^{\prime}}{\delta d}=\lambda_{2} \\
& \frac{\delta d^{\prime}}{\delta d}=\frac{1-\frac{d}{h}}{\left(\frac{d^{\prime}}{h}\right)^{2} \kappa \cdot h-\frac{d^{\prime}}{h}(2 \cdot \kappa \cdot h+1)+1+\kappa \cdot h}
\end{aligned}
$$

This differential equation determines the position $d^{\prime}$ of a packet of rubber, and therefore its deformation.

Strain energy distribution. To analyze the strain energy, the differential equation was solved numerically. The boundary conditions were set on the outer boundary of the hull at $d^{\prime}=0$ to $\lambda_{2}=1$ and $\lambda_{3}=\frac{1}{\lambda_{1}}$.

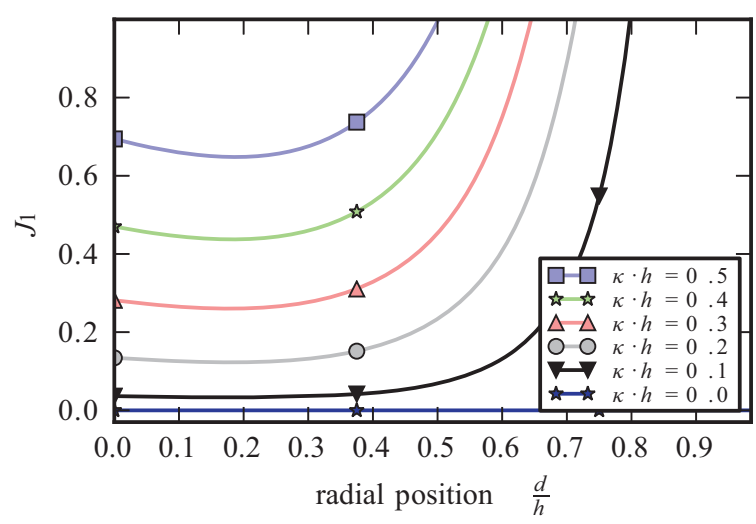

Fig. 21. Strain energy density distribution within the rubber hull for different normalized curvatures.

Figure 21 shows the strain energy density with respect to the normalized depth $\frac{d}{h}$ within the hull and at different normalized curvatures $\kappa \cdot h$. We can see that the strain energy density stays surprisingly flat even for moderately thick hulls and at very strong curvatures.

The reason for this surprising result can be understood when looking at the principal stretches when the rubber moves outwards radially (thinning the wall). Circumferential stretch $\lambda_{3}$ increases, but at the same time radial stretch $\lambda_{2}$ decreases as the packet gets more compressed. Also $\lambda_{1}$ decreases with the distance from the bottom layer.

The analysis shows that as long as the hull thickness is less than half the actuator height, we can assume a uniform strain energy distribution for modeling. Staying below this limit also avoids material fatigue of the rubber on the inside of the air chamber.

\section{B.7 Justifying simplification: Neo-Hookean deformation model}

An alternative to the simple neo-Hookean deformation model used in our model is the generalized Mooney-Rivlin model for incompressible hyperelastic materials. It states a polynomial approximation of strain energy density, using the strain tensor invariants:

$$
\frac{d W}{d v}=\sum_{i, j=0}^{n} C_{i j} \cdot J_{1}^{i} \cdot J_{2}^{j}
$$

Silicone rubbers have rather small coefficients for $C_{01}$, $C_{02}$ and $C_{20}$ though. The coefficients published by Meier et al. (2005) have the following relations:

$$
\begin{aligned}
& C_{01} \approx \frac{1}{50} \cdot C_{10} \\
& C_{20} \approx \frac{1}{500} \cdot C_{10} \\
& C_{02} \approx 0
\end{aligned}
$$




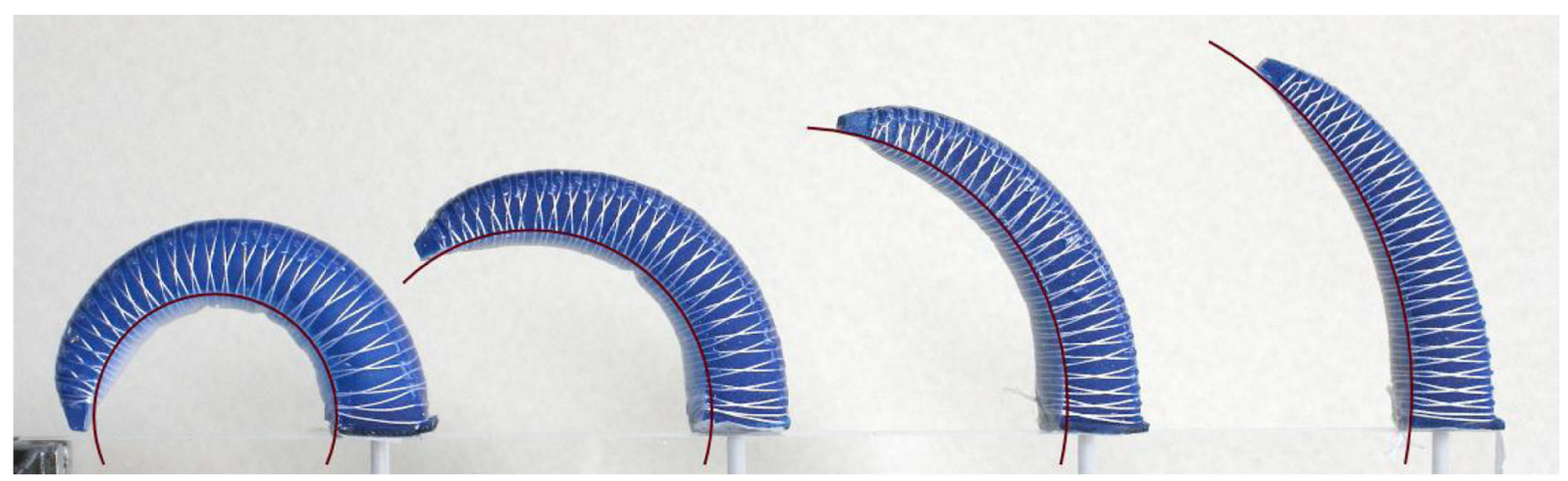

Fig. 22. Example of four fingers with different actuation ratios, inflated to $46.3 \mathrm{kPa}$. The overlaid circle segments indicate the constant curvature along the bottom layer.

Ignoring the coefficients $C_{01}, C_{02}$ and $C_{20}$ (i.e. setting them to 0 ) yields the neo-Hookean model.

We can bound the error to strain energy when assuming a maximum stretch of $\lambda_{1}<3$ (which relates to an actuator bending at the radius of half its height), which given Equation (5) bounds the strain tensor values to

$$
\begin{aligned}
& J_{1}<7.11 \\
& J_{2}<7.11
\end{aligned}
$$

The terms dropped from the Mooney-Rivlin model are bounded to

$$
\begin{aligned}
& C_{01} \cdot J_{2}<0.02 \cdot C_{10} \cdot J_{1} \\
& C_{20} \cdot J_{1}^{2}<0.014 \cdot C_{10} \cdot J_{1}
\end{aligned}
$$

which results in a total error of less than 3.4\%.

\section{B.8 Justifying simplification: Ignore material stiffening}

Elastomers exhibit a stiffening at large stretches. This is modeled by augmenting the strain energy function with an additional parameter. The Gent model (Gent, 2012) augments the strain energy function with a logarithmic term:

$$
\begin{aligned}
W & =\frac{G}{2} \cdot J_{1}\left(\lambda_{\max }\right) \ln \left(1-\frac{J_{1}\left(\lambda_{1}\right)}{J_{1}\left(\lambda_{\max }\right)}\right) \cdot V_{\text {sil }} \\
& =\frac{G}{2} \cdot J_{m} \ln \left(1-\frac{J_{1}}{J_{m}}\right) \cdot V_{\text {sil }}
\end{aligned}
$$

With the additional material parameter $\lambda_{\max }$ which is the stretch where the material exhibits unlimited stiffness.

The rubber used for the PneuFlex actuator typically has $\lambda_{\max } \approx 10$, which results in

$$
J_{m} \approx 100
$$

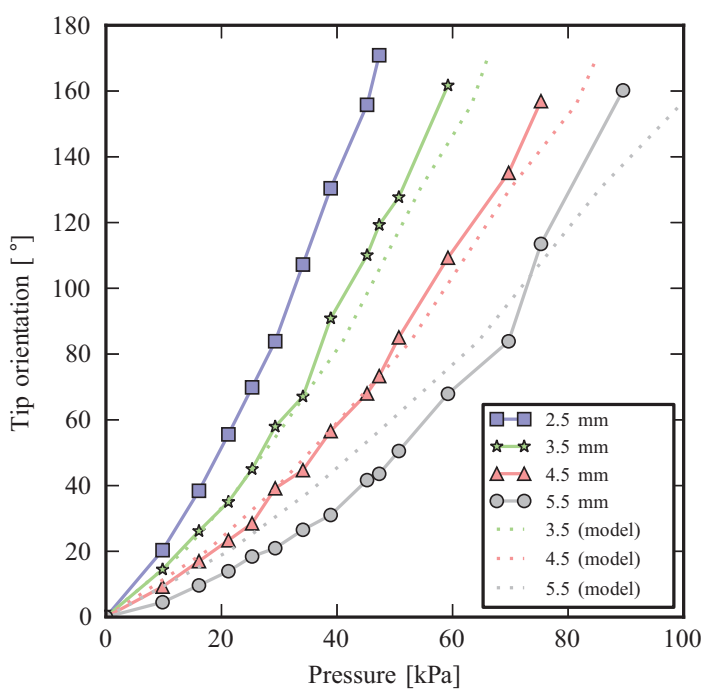

Fig. 23. Tip orientation versus inflation pressure for different rubber hull thicknesses. Dotted lines indicate model estimates based on the $2.5 \mathrm{~mm}$ measurement.

When computing the strain energy gradient using the Gent model, we obtain

$$
\begin{aligned}
\frac{\delta W_{\text {sil }}^{\text {Gent }}}{\delta \lambda_{1}} & =C_{1} \cdot J_{m} \cdot V_{\text {sil }} \cdot \frac{1}{1-\frac{\lambda_{1}^{2}+\lambda_{1}^{-2}-2}{J_{m}}} \cdot \frac{2 \lambda_{1}-2 \cdot \lambda_{1}^{-3}}{J_{m}} \\
& =\frac{1}{1-\frac{\lambda_{1}^{2}+\lambda_{1}^{-2}-2}{J_{m}}} \cdot \frac{\delta W_{\text {sil }}}{\delta \lambda_{1}}
\end{aligned}
$$

For plausible values of $\lambda_{1}<3$ and $J m=100$, the ignored stiffening factor can be bounded to

$$
\frac{\delta W_{\text {sil }}^{\text {Gent }}}{\delta \lambda_{1}}<1.077 \cdot \frac{\delta W_{\text {sil }}}{\delta \lambda_{1}}
$$

So ignoring the material stiffening introduces an error of less than $7.7 \%$. 


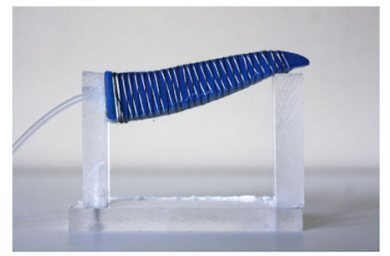

(a) $0 \mathrm{kPa}$

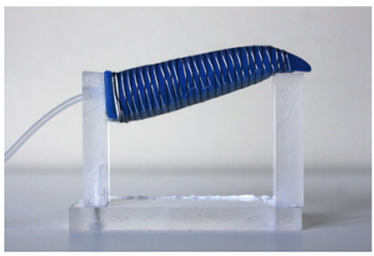

(b) $17.3 \mathrm{kPa}$

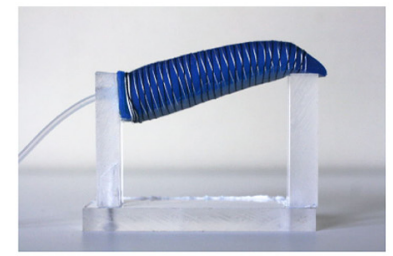

(c) $41.6 \mathrm{kPa}$

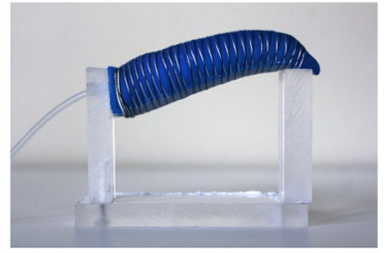

(d) $80.1 \mathrm{kPa}$

Fig. 24. The finger is blocked by a fingertip contact while being inflated. An actuator with a linearly decreasing stiffness profile will show a constant curvature along the actuator under such load.

\section{B.9 Justifying simplification: Ignoring the side walls}

For analyzing the impact of ignoring the side walls, we can conceptually split the cross section of a real PneuFlex actuator into many small parts, of which each behaves according to the simple model.

As there is no air below the side walls (only more rubber), there is no additional force applied by gas pressure.

The amount of deformation work $\frac{\delta W_{\text {sil }}}{\delta \lambda_{1}}$ does increase, but the $J_{1}$ also drops off quadratically when approaching the bottom layer. By using a constant ratio between the thickness of the side wall and the top side of the actuator, the error being made when computing the actuation ratio can be made constant.

For the stiffness, the side walls play even less of a role, as it scales with $h^{3}$.

\section{B.10 Experimental validation}

To validate the design rules we developed, we conducted two experiments. The first one validates the equation for the actuation ratio, the second the equation for actuator stiffness.

\section{B.10.1 Actuators with varying $d$}

The model predicts that the actuation ratio is only dependent on hull thickness $d$ and the shear modulus of the rubber. To test this, we built four actuators with the same shape but with a $d$ of $2.5 \mathrm{~mm}, 3.5 \mathrm{~mm}, 4.5 \mathrm{~mm}$ and $5.5 \mathrm{~mm}$, respectively.

Figure 22 shows the actuators at a pressure of $46.3 \mathrm{kPa}$. Despite the change in height and width, the curvatures of the bottom layers are constant along the actuators, as indicated by the circle segments. We can therefore validate the model's prediction that height and width do not influence actuation ratio. Please note that the circle segments are placed on top of the edges of the bottom layers, where the fibers of the embedded fabric are most stressed.

Figure 23 shows the relationship between pressure and fingertip orientation, which is an aggregated measure of the curvature along the actuator. The curves for each actuator show the nonlinear behavior predicted by Equation (13), i.e. an increase in actuation ratio towards higher curvatures.

The dotted lines in Figure 23 indicate the actuation ratio when scaling the measurements of the thinnest actuator $(2.5 \mathrm{~mm})$ to the thickness of the other actuators according to our model. Model and measurement agree well for $3.5 \mathrm{~mm}$ and $4.5 \mathrm{~mm}$. At $5.5 \mathrm{~mm}$ it is clearly visible that the nonlinearity of the actuation ratio increases, making the actuator stiffer than expected at low pressures.

\section{B.10.2 Actuator with linear stiffness}

To validate Equations (11) and (15), we can apply a force at the tip of an actuator. The actuator has a constant actuation ratio along its main axis. The contact force will create a bending moment to segments of the actuator that increases linearly with the segment's distance from the contact point. At small curvatures, i.e. an almost straight finger, the distance along the bottom layer will approximate the Euclidean distance well. For a straight actuator, we can therefore assume the moment along the actuator to be increasing linearly. If the actuator has a linear stiffness profile along the actuator and a constant actuation ratio too, then the load moment and actuated moment will cancel out and yield a constant curvature. The constant actuation ratio is demonstrated in Figure 22.

Figure 24 shows an actuator at four different pressures. The curvature stays constant during a large range of inflation pressures, with the highest pressure corresponding to about a $360^{\circ}$ rotation if there was no contact. The actuator therefore has a linear stiffness profile, which validates Equation (11).

The upwards rotation of the whole finger relative to the fixture is caused by the rubber cap which closes the air chamber at the base of the finger. This also causes the small curvature changes between different pressures. Figure 24(d) shows a slightly stronger curvature close to the tip. This may be caused by difference between contact point location and the point where the stiffness profile reaches zero stiffness, which is exactly at the tip. 


\section{B.11 Summary}

The presented theoretic model yields two simple design rules (Equations (14) and (15)) for designing actuation ratios and stiffnesses along a PneuFlex actuator. We also validated the scaling behavior of the model in two experiments.

Based on the analysis, we can give the following recommendations for choosing geometric parameters.
- Active layer thickness should stay less than half the actuator height: $\frac{d}{h}<0.5$.

- To achieve a minimal bending radius of 1.5 times actuator height, the rubber should have an elongation at break of at least $500 \%$.

- Material stiffening can usually be ignored with silicone rubber, the error is typically less than $7.7 \%$. 